\title{
NOMINALISIERUNGEN ALS BEISPIEL GESCHWÄCHTER SYNTAKTISCHER KONSTRUKTIONEN ${ }^{1}$
}

\section{ZUM THEORETISCHEN HINTERGRUND DER ARBEIT}

In unserer Arbeitshypothese postulieren wir die Existenz von einerseits verstärkten und entsprechenden nichtverstärkten syntaktischen Konstruktionen und andererseits die Existenz von geschwächten und entsprechenden nichtgeschwächten syntaktischen Konstruktionen.

Unsere Grundannahme besagt folgendes: Am Anfang (d.h. im Stadium, in welchem eine Konstruktion nur als eine syntaktische Variante eines anderen Ausdrucks zu werten und noch nicht grammatikalisiert ist) behaupten sich verstärkte Konstruktionen vorzugsweise unter relativ komplizierten (markierten) grammatischen Verhältnissen und verbreiten sich in ihrer späteren Entwicklung unter Umständen auch unter weniger komplizierten grammatischen Verhältnissen. Geschwächte Konstruktionen dagegen behaupten sich am Anfang vorzugsweise unter relativ einfachen (weniger markierten) grammatischen Verhältnissen und verbreiten sich später möglicherweise auch unter weniger einfachen grammatischen Verhältnissen. ${ }^{2}$

\section{DIE ARBEITSHYPOTHESE DER UNTERSUCHUNG}

Die Arbeitshypothese unserer Untersuchung über die Nominalisierungen in der deutschen Gegenwartssprache basiert

1 Diese Untersuchung ist am 19.7.1990 vor der Kommission Prof. Dr. Janez Orešnik, Prof. Dr. Stanko Žepić und Doz. Dr. Anton Janko verteidigt und an der Philosophischen Fakultät in Ljubljana als Magisterarbeit angenommen worden. Besonderen Dank schulde ich meinem Berater Professor Dr. Janez Orešnik, der diese Arbeit angeregt hat und immer die Zeit fand, mich mit Hinweisen und fachlicher Kritik zu unterstïtzen.

2 Näheres zum theoretischen Hintergrund dieser Untersuchung und einiger anderer Arbeiten ist in Orešnik/Snedec/Teržan/Trobevšek-Drobnak (1990) und in Orešnik (1990b) zu finden. 
1.1 auf der Annahme, daß der Gliedkern einer Nominalisierung (d.h. eine deverbale Ableitung, ein Verbalabstraktum) im Vergleich zu seiner verbalen Basis eine verstärkte Wortbildungskonstruktion ist,

1.2 auf der Annahme, daß eine Nominalisierung im Vergleich zum entsprechenden Satz mit finiter Verbform eine geschwächte syntaktische Konstruktion ist,

1.3 auf der Annahme, daß bestimmte Werte grammatischer Parameter in der Sprache relativ einfach (unmarkiert) und bestimmte Werte grammatischer Parameter in der Sprache relativ kompliziert (markiert) sind (Orešnik/Snedec/Teržan/Trobevšek-Drobnak 1990:78, Orešnik 1990b:87-88),

1.4 auf der Annahme, daß sich geschwächte syntaktische Konstruktionen am Anfang ihrer Entwicklung unter relativ einfachen (unmarkierten bzw. weniger markierten) grammatischen Verhältnissen behaupten (Orešnik/Snedec/Teržan/Trobevšek-Drobnak 1990:8, Orešnik 1990b:87), und

1.5 auf der Annahme, daß eine geschwächte syntaktische Konstruktion häufiger vorkommt als die entsprechende nichtgeschwächte Konstruktion (Orešnik/Snedec/ Teržan/Trobevšek-Drobnak 1990:8, Orešnik 1990b:90-91).

\section{DIE VORHERSAGEN}

Der Kern einer Nominalisierung (ein Verbalabstraktum) ist im Vergleich zu seiner verbalen Basis eine verstärkte Form. Diese Behauptung wird folgendermaßen begründet:

Dem Verbalstamm wird beim Ableitungsvorgang meist ein (oder sogar mehr als ein) Morphem hinzugefügt. Der Kern einer Nominalisierung ist demnach komplizierter gebaut als der entsprechende Verbstamm:

z.B. (2.1) Verwandl + ung.

Der Kern einer Nominalisierung behält meist nur bestimmte Bedeutungsvarianten der verbalen Basis bei und hat daher meistens weniger Bedeutungsvarianten als das zugrundeliegende Verb:

z.B. (2.2) erhalten : Erhaltung.

Verbalabstrakta sind überwiegend von "schweren" Verben abgeleitet, d.h. die zugrundeliegenden Verben sind kompliziertere Morphemkonstruktionen, daher längere Wörter, kommen relativ selten vor und sind vorwiegend Vollverben:

z.B. (2.3) sich einpassen ( $\rightarrow$ die Einpassung).

Hinsichtlich ihrer Bedeutung gehören die Kerne der Nominalisierungen (die Verbalabstrakta) zu den kompliziertesten Substantiven. Nach Mayerthaler (1980:17f) sind dynamische Abstrakta komplizierter als statische Abstrakta und letztere komplizierter als Konkreta): 
z.B. (2.4) Hans < Tisch < Liebe < Überschreitung.

Die Vorhersagen für die Verben, die den Nominalisierungskernen zugrundeliegen, im Vergleich zu den Vollverben in den Sätzen des Kontrollsamples: ${ }^{3}$

2.1 Die zugrundeliegenden Verben der Nominalisierungskerne sind häufiger komplizierte Wortbildungskonstruktionen, d.h. präfigierte, suffigierte und zusammengesetzte Verben.

2.2 Die zugrundeliegenden Verben der Nominalisierungskerne sind häufiger polysegmental, d.h. sie bestehen häufiger aus drei, vier, fünf oder mehr Silben.

2.3 Die zugrundeliegenden Verben der Nominalisierungskerne kommen seltener vor, d.h. die Häufigkeit der morphologischen Formen eines zugrundeliegenden Verbs ist nach Kaedings Häufigkeitswörterbuch (in: Meier 1978, zweiter Band, 3-111) kleiner als 1000 .

Die Vorhersage, daß die zugrundeliegenden Verben der Nominalisierungskerne häufiger Vollverben als Hilfsverben sind, wurde nicht mit Hilfe des Korpus geprüft, da die Zahl der gefundenen Nominalisierungskerne, denen ein Hilfsverb zugrundelag, bei weitem zu gering war, als daß ein statistischer Vergleich sinnvoll gewesen wäre.

Die Vorhersagen für das Verhalten der Nominalisierungskerne im Vergleich zu jenen Substantiven im Kontrollsample, die keine deverbalen Ableitungen sind:

2.4 Nominalisierungskerne sind häufiger komplizierte Morphemkonstruktionen, d.h. sie sind häufiger präfigiert, suffigiert oder zusammengesetzt.

2.5 Nominalisierungskerne kommen seltener vor, d.h. die Häufigkeit der morphologischen Formen eines Substantivs ist nach Kaedings Häufigkeitswörterbuch (in: Meier 1978, zweiter Band, 3-111) kleiner als 1000.

Die Vorhersage für das Verhalten der Nominalisierungskerne im Vergleich zu ihren zugrundeliegenden Verben:

2.6 Nominalisierungskerne sind häufiger bedeutungsenger als ihre zugrundeliegenden Verben, d.h. die Zahl ihrer Bedeutungsvarianten ist laut Wörterbuch (Duden 1976-81) kleiner.

Die Vorhersage, daß die Nominalisierungskerne häufiger eine dynamische als eine statische Bedeutung haben, wurde nicht statistisch geprüft, da in relativ vielen Fällen nicht mit ausreichender Sicherheit festgestellt werden konnte, ob eine dynamische oder eine statische Bedeutungsvariante des betreffenden Verbalabstraktums vorliegt.

Eine Nominalisierung ist im Vergleich zum entsprechenden Satz mit finiter Verbform eine geschwächte syntaktische Konstruktion, weil die syntaktischen Beziehungen zwischen den Teilen einer Nominalisierung weniger deutlich sind als die entsprechenden syntaktischen Beziehungen zwischen den einzelnen Teilen des entsprechenden Satzes mit finter Verbform. Während eine finite Verbform über Morpheme ver- 
fügt, mit denen man die Kategorien Person, Numerus, Tempus, Modus und Genus verbi zum Ausdruck bringen kann, fehlen einer Nominalisierung solche Morpheme. Eine finite Verbform fordert überdies die Nennung einer bestimmten Anzahl von (obligatorischen) Ergänzungen, wohingegen die Ergänzungen der Nominalisierungen, die den Status von Attributen haben, grundsätzlich fakultativ sind und daher fehlen dürfen. Nominalisierungen können außerdem die Realisierung nebensatzeinleitender Konjunktionen ersparen.

z.B. (2.5a) Ich mache Sie für die Einhaltung verantwortlich. (Fische 191/1)

(2.5b) Ich mache Sie dafür verantwortlich, daß die Soldaten den Befehl einhalten /

daß der Befehl eingehalten wird.

Die Vorhersage für die (syntaktische Konstruktion der) Nominalisierungen im Vergleich zu den Sätzen mit den zugrundeliegenden Verben:

2.7 Nominalisierungen kommen häufiger ohne adnominale Ergänzungen vor. Dabei ist die Zahl der adnominalen Ergänzungen in Nominalisierungen kleiner als die Zahl der verbalen Ergänzungen in Sätzen mit den entsprechenden zugrundeliegenden Verben.

Die Vorhersagen für die (syntaktische Konstruktion der) Nominalisierungen im Vergleich zu den Substantivgruppen mit Satzgliedcharakter in den Sätzen des Kontrollsamples:

2.8 Wenn in der Nominalisierung eine adnominale Ergänzung auftritt und sie etwas Belebtes bezeichnet, handelt es sich meistens um das Subjekt des zugrundeliegenden Verbs.

2.9 Wenn in der Nominalisierung eine adnominale Ergänzung auftritt und sie etwas Unbelebtes bezeichnet, handelt es sich meist um das direkte Objekt des zugrundeliegenden Verbs.

2.10 Eine Nominalisierung bezeichnet etwas Unbelebtes und kommt daher im Satz häufiger als direktes Objekt vor und seltener als anderes Satzglied (Das direkte Objekt ist das einfachste Satzglied für Unbelebtes).

2.11 Eine Nominalisierung tritt häufiger als Thema eines Satzes auf und seltener als Rhema (Das Thema gilt im Vergleich zum Rhema als weniger kompliziert). ${ }^{4}$

Eine Nominalisierung ist im Vergleich zum entsprechenden Satz mit finiter Verbform eine geschwächte syntaktische Konstruktion. Daher treten sie häufiger in einfacherem (weniger markierten) Kontext auf und seltener in komplizierterem (markierterem) Kontext (zur Markiertheit grammatischer Parameter vgl. Orešnik/Snedec/Teržan/Trobevšek-Drobnak 1990:7-8 oder Orešnik 1990b:87-88).

4. Bezüglich der Thema-Rhema-Verteilung wurden nur Substantivgruppen miteinander verglichen, die als Gliedkern ein Substantiv enthielten (also nicht pronominale Gliedkerne). 
Die Vorhersagen zur Kompliziertheit des Kontextes der Nominalisierungen im Vergleich zur Kompliziertheit der Sätze und satzwertigen Konstruktionen ${ }^{5}$ im Kontrollsample:

2.12 Nominalisierungen kommen häufiger in Hauptsätzen und seltener in Nebensätzen vor.

2.13 Nominalisierungen treten häufiger in abhängigen Sätzen mit infiniter Verbform auf als in abhängigen Sätzen mit finiter Verbform. ${ }^{6}$

2.14 Nominalisierungen kommen häufiger in Aussagesätzen und seltener in anderen Satzarten vor.

2.15 Nominalisierungen treten häufiger in bejahenden Sätzen als in verneinenden Sätzen auf.

2.16-2.20 Nominalisierungen kommen häufiger vor in Sätzen mit der Verbform in der dritten Person, im Singular, im Präsens, im Indikativ und im Aktiv.

\section{DIE ANALYSE DER TEXTE IM KORPUS}

In diesem Abschnitt soll kurz dargelegt werden, wie das ausgesuchte Sprachmaterial im Hinblick auf unsere Aufgabenstellung bearbeitet worden ist.

\section{KRITERIEN FÜR DIE AUSWAHL VON NOMINALISIERUNGEN}

GRUNDSAMPLE 'A' (Datenbasis: "Die Fische"):

3.1 Verbalsubstantive mit verschiedenen Affixen;

3.2 Verbalsubstantive mit abstrakter (nichtkonkreter) Bedeutung:

a) Geschehensbezeichnungen (Vorgänge, Handlungen)

b) Zustandsbezeichnungen

c) Ergebnisbezeichnungen;

3.3 Verbalsubstantive,

a) die Bestandteil eines Funktionsverbgefüges ("FVG") sind

b) die zusammen mit dem Satzverb eine freie Fügung ("FF") bilden.

5 Als Nebensätze gelten in dieser Untersuchung abhängige Sätze mit finitem Verb an erster, zweiter und letzter Stelle. Mit satzwertigen Konstruktionen sind Infinitivsätze und Partizipialkonstruktionen gemeint.

6 In Infinitivsätzen oder satzwertigen Partizipialkonstruktionen sind die syntaktischen Beziehungen zwischen den einzelnen Teilen weniger explizit als in abhängigen Sätzen mit finiter Verbform. Vgl. aber Mayerthaler/Fliedl/Winkl 1991, die Infinitivkonstruktionen als markierter ansehen. 
GRUNDSAMPLE 'B' (Datenbasis: "Die Fische", "Meinung gegen Meinung. Fragen der Verkehrssicherheit", "Schulklassengespräch mit Günter Grass", "Moral 71. Zum Beispiel Abtreibung"):

\subsection{Verbalsubstantive mit verschiedenen Affixen;}

3.5 Verbalsubstantive mit abstrakter (nichtkonkreter) Bedeutung:

a) Geschehensbezeichnungen (Vorgänge, Handlungen)

b) Zustandsbezeichnungen;

3.6 Verbalsubstantive, die zusammen mit dem Satzverb eine freie Fügung ("FF") bilden und nicht als Funktionsnomen anzusehen sind;

3.7 Verbalsubstantive, die die syntaktische Valenz ihrer Basisverben nach solchen Regeln übernehmen, die auch für nichtlexikalisierte Verbalsubstantive auf [en] gelten (vgl. ten Cate 1985:172ff, Schäublin 1972:22ff, Teubert 1979:79f; vgl. auch Helbig 1986);

3.8 Verbalsubstantive,

a) die im Text in der Singularform auftreten,

b) die nicht pluralisierbar sind (vgl. ten Cate 1985:214ff);

3.9 Verbalsubstantive mit derselben Betonung wie das Basisverb (vgl. ten Cate 1985:156f).

\section{BEISPIELE AUS DEM GRUNDSAMPLE ' $\mathbf{A}^{7}$}

\section{GESCHEHENSBEZEICHNUNGEN OHNE ATTRIBUTIVE ERGÄNZUNGEN:}

(3.1) Ich mache Sie für die EINHALTUNG verantwortlich. $(191 / 1)^{8}$

- zugrundeliegendes Verb des Verbalabstraktums:

7 Erklärung der Abkürzungen: sub $=$ Subjekt, akk = Akkusativergänzung (Akkusativobjekt, direktes Objekt), gen $=$ Genitivergänzung, dat $=$ Dativergänzung, prp $=$ Präpositionalergänzung, adv $=$ Adverbialergänzung. Steht eine dieser Abkürzungen in runden Klammern, bedeutet dies, daß es sich um eine fakultative Ergänzung handelt. Die Abkürzung sub $\ll$ akk bedeutet, daß die Akkusativergänzung eines Aktivsatzes, wenn dieser in einen Satz mit dem Sein-Passiv umgeformt wird, als Subjekt auftritt bzw. verstanden wird. Eine durchgestrichene Abkürzung für eine Ergänzung (z.B. sub) steht dann, wenn ein Argument, das Verb und Verbalabstraktum gemeinsam ist, nicht als adnominale Ergänzung realisiert werden kann. Das Zeichen - bedeutet, daß eine Erscheinung nicht notiert werden konnte. W-Passiv bedeutet Werden-Passiv, S-Passiv Sein-Passiv, Infin. Infinitivsatz und Part. Partizipialphrase (Partizipialkonstruktion). Die Abkürzung tanim bedeutet Belebtes, -anim hingegen Unbelebtes.

8 In den Beispielen aus den beiden Grundsamples werden die Verbalabstrakta mit Großbuchstaben geschrieben, die Ergänzungen des Verbalabstraktums kursiv gedruckt und die gesamte Nominalisierung(sphrase) unterstrichen. Bei Funktionsverbgefügen werden auch die Funktionsverben unterstrichen. Zu den Zahlenangaben in runden Klammern nach einem Beispiel: die erste Zahl steht für die Seitenzahl in der verwendeten Textausgabe, die zweite Zahl hingegen ist die fortlaufende Nummer der Nominalisierung auf der Seite im Text. 
Wortbildung: kompliziert, Länge: lang (3 Silben),

Häufigkeit: unklar.

- Verbalabstraktum der Nominalisierung:

Wortbildung: kompliziert, Häufigkeit: selten,

Bedeutungsvarianten: weniger, Bedeutung: dynamisch.

- Nominalisierung:

gemeinsame Ergänzungen Verb/Substantiv: sub akk, ${ }^{9}$

Zahl der Ergänzungen der Nominalisierung: 0, ${ }^{10}$

Zahl der Ergänzungen des Verbs minimal: 1 (W-Passiv), ${ }^{11}$

Zahl aller Ergänzungen des Verbs: 2 (Aktiv),

Zahl der adnominalen Ergänzungen kleiner: ja, ${ }^{12}$

Subjekt (sub) des Verbs als Attribut: nein, ${ }^{13}$

Direktes Objekt (akk) des V. als Attribut: nein, ${ }^{14}$

Nominalisierung als direktes Objekt (akk): nein,

Nominalisierung als Thema des Satzes: nein, ${ }^{15}$

9 Das Verbalabstraktum und das zugrundeliegende Verb verfügen über eine bestimmte Wertigkeit (Valenz). In ihrem Stellenplan gibt es bestimmte Leerstellen, die von Ergänzungen besetzt werden müssen bzw. können. Adnominale Ergänzungen sind im Gegensatz zu verbalen in der Regel fakultativ. Die Valenzanalyse folgt im wesentlichen Engel 1988, Engel/Schumacher 1978, Latour 1985 und Sommerfeldt/ Schreiber 1983. Zum Begriff 'Argumentvererbung' siehe Toman 1987.

10 Evidentiert wird die Zahl der als Attribute auftretenden Ergänzungen des Verbalabstraktums im entsprechenden Satz aus dem Grundsample.

11 Die "Zahl der Ergänzungen des Verbs minimal" ergibt sich, wenn man eine Nominalisierung auf einen Satz zurückführt (vgl. das Beispiel (2.5ab)). Dieser Satz wird in die Umgebung der Nominalisierung (d.h. in den Matrixsatz anstelle der Nominalisierung) eingebettet. Je nach Umgebung erhält dieser Konstituentensatz die Form eines aktivischen oder passivischen Nebensatzes oder die Form eines Infinitivsatzes oder einer Partizipialkonstruktion. In vielen Fällen erlaubt der Matrixsatz verschiedene Formen des Konstituentensatzes. Für jede Form eines Konstituentensatzes wurde festgestellt, wieviele Ergänzungen (mit Rücksicht auf den Matrixsatz) darin obligatorisch sind. Vgl. auch die nächste Fußnote.

12 Verglichen wurde mit der kleineren Zahl der verbalen Ergänzungen. Meist handelte es sich dabei um die Zahl der Ergänzungen des betreffenden Verbs im Passiv (wenn dieser vom betreffenden Verb gebildet werden konnte und der Matrixsatz des Grundsamples diese Form des Konstituentensatzes nahelegte) oder häufig auch um die Zahl der Ergänzungen des betreffenden Verbs in einem Infinitivsatz (wenn dieser durch die Form des Matrixsatzes nahegelegt wurde). Waren sowohl ein finiter wie ein infiniter Konstituentensatz gleichermaßen wahrscheinlich, wurde lediglich die Möglichkeit des finiten Konstituentensatzes eingetragen und zum Vergleich herangezogen.

13 Solche Attribute werden auch als 'Subjektgenitiv' bezeichnet.

14 Solche Attribute werden auch 'Objektgenitiv' genannt.

15 Als Thema eines Satzes wurde bei normaler (d.h. bei nicht-kontrastierender oder nicht-emphatischer) Intonation in der Regel das erste Satzglied, als Rhema (rheme proper) hingegen in der Regel das letzte und/oder Hauptbetonung tragende Satzglied betrachtet. Satzglieder, die in einer Voranalyse der Transitionsphase zugerechnet wurden, sind in diesem Aufsatz allerdings der Einfachheit halber ebenfalls als Rhema gewertet worden, da in der Voranalyse hinsichtlich der 
- Kontext der Nominalisierung:

Hauptsatz: ja, Aussagesatz: ja, affirmativ: ja,

3. Person: nein, Singular: ja, Präsens: ja,

Indikativ: ja, Aktiv: ja.

(3.2) Auf diesen SIEG muß es leider verzichten. (189/8)

- zugrundeliegendes Verb des Verbalabstraktums:

Wortbildung: einfach, Länge: kurz (2 Silben),

Häufigkeit: selten.

- Verbalabstraktum der Nominalisierung:

Wortbildung: einfach, Häufigkeit: selten,

Bedeutungsvarianten: gleich, Bedeutung: dynamisch.

- Nominalisierung:

gemeinsame Ergänzungen Verb/Substantiv: sub (prp),

Zahl der Ergänzungen der Nominalisierung: 0,

Zahl der Ergänzungen des Verbs minimal: 0 (Infin.),

Zahl aller Ergänzungen des Verbs: 2 (Aktiv),

Zahl der adnominalen Ergänzungen kleiner: nein,

Subjekt (sub) des Verbs als Attribut: nein,

Direktes Objekt (akk) des V. als Attribut: nein,

Nominalisierung als direktes Objekt (akk): nein,

Nominalisierung als Thema des Satzes: ja.

- Kontext der Nominalisierung:

Hauptsatz: ja, Aussagesatz: ja, affirmativ: ja,

3. Person: ja, Singular: ja, Präsens: ja,

Indikativ: ja, Aktiv: ja.

\section{GESCHEHENSBEZEICHNUNGEN MIT ATTRIBUTIVEN ERGÄNZUNGEN:}

(3.3) Bei Leichenöffnungen, sehen Sie, erspart ein kleiner Kosthappen oft langwieriges MIKROSKOPIEREN der Gewebe. (200/3)

- zugrundeliegendes Verb des Verbalabstraktums:

Wortbildung: kompliziert, Länge: lang (5 Silben),

Häufigkeit: selten.

- Verbalabstraktum der Nominalisierung:

Wortbildung: kompliziert, Häufigkeit: selten,

Bedeutungsvarianten: unklar, Bedeutung: dynamisch.

- Nominalisierung:

gemeinsame Ergänzungen Verb/Substantiv: sub akk,

Satzglieder in der Transitionsphase keine signifikanten Unterschiede zwischen Grund- und Kontrollsample festgestellt werden konnten. 
Zahl der Ergänzungen der Nominalisierung: 1, Zahl der Ergänzungen des Verbs minimal: 1 (Infin.),

Zahl aller Ergänzungen des Verbs: 2 (Aktiv),

Zahl der adnominalen Ergänzungen kleiner: nein,

Subjekt (sub) des Verbs als Attribut: nein,

Direktes Objekt (akk) des V. als Attribut: ja (-anim),

Nominalisierung als direktes Objekt (akk): ja,

Nominalisierung als Thema des Satzes: nein,

- Kontext der Nominalisierung:

Hauptsatz: ja, Aussagesatz: ja, affirmativ: ja,

3. Person: ja, Singular: ja, Präsens: ja,

Indikativ: ja, Aktiv: ja.

(3.4) Sie hören nicht auf mein GESCHWÄTZ, nein ? (212/5)

- zugrundeliegendes Verb des Verbalabstraktums:

Wortbildung: einfach, Länge: kurz (2 Silben),

Häufigkeit: selten.

- Verbalabstraktum der Nominalisierung:

Wortbildung: kompliziert, Häufigkeit: selten,

Bedeutungsvarianten: weniger, Bedeutung: dynamisch.

- Nominalisierung:

gemeinsame Ergänzungen Verb/Substantiv: sub (prp),

Zahl der Ergänzungen der Nominalisierung: 1,

Zahl der Ergänzungen des Verbs minimal: 1 (Aktiv),

Zahl aller Ergänzungen des Verbs: 2 (Aktiv),

Zahl der adnominalen Ergänzungen kleiner: nein,

Subjekt (sub) des Verbs als Attribut: ja (+anim),

Direktes Objekt (akk) des V. als Attribut: nein,

Nominalisierung als direktes Objekt (akk): nein,

Nominalisierung als Thema des Satzes: nein,

- Kontext der Nominalisierung:

Hauptsatz: ja, Aussagesatz: ja, affirmativ: nein,

3. Person: ja, Singular: nein, Präsens: ja,

Indikativ: ja, Aktiv: ja.

(3.5) Meine Herren, ich übernehme die AUSSCHAU nach den Dragonern. (215/2)

- zugrundeliegendes Verb des Verbalabstraktums:

Wortbildung: kompliziert, Länge: lang (3 Silben),

Häufigkeit: selten.

- Verbalabstraktum der Nominalisierung:

Wortbildung: einfach, Häufigkeit: selten,

Bedeutungsvarianten: weniger, Bedeutung: dynamisch. 


\section{- Nominalisierung:}

gemeinsame Ergänzungen Verb/Substantiv: sub prp,

Zahl der Ergänzungen der Nominalisierung: 1,

Zahl der Ergänzungen des Verbs minimal: 1 (Infin.),

Zahl aller Ergänzungen des Verbs: 2 (Aktiv),

Zahl der adnominalen Ergänzungen kleiner: nein,

Subjekt (sub) des Verbs als Attribut: nein,

Direktes Objekt (akk) des V. als Attribut: nein,

Nominalisierung als direktes Objekt (akk): ja,

Nominalisierung als Thema des Satzes: nein,

- Kontext der Nominalisierung:

Hauptsatz: ja, Aussagesatz: ja, affirmativ: ja,

3. Person: nein, Singular: ja, Präsens: ja,

Indikativ: ja, Aktiv: ja.

\section{ZUSTANDSBEZEICHNUNGEN OHNE ATTRIBUTIVE ERGÄNZUNGEN:}

(3.6) Wir versuchen, die beim Fang auftretende ERREGUNG auf den kleinsten Grad herabzusetzen. (191/4)

- zugrundeliegendes Verb des Verbalabstraktums:

Wortbildung: kompliziert, Länge: lang (3 Silben),

Häufigkeit: selten.

- Verbalabstraktum der Nominalisierung:

Wortbildung: kompliziert, Häufigkeit: selten,

Bedeutungsvarianten: weniger, Bedeutung: statisch.

- Nominalisierung:

gemeinsame Ergänzungen Verb/Substantiv: sub $<<$ akk,

Zahl der Ergänzungen der Nominalisierung: 0,

Zahl der Ergänzungen des Verbs minimal: 1 (S-Passiv),

Zahl aller Ergänzungen des Verbs: 2 (Aktiv),

Zahl der adnominalen Ergänzungen kleiner: ja,

Subjekt (sub) des Verbs als Attribut: nein,

Direktes Objekt (akk) des V. als Attribut: nein,

Nominalisierung als direktes Objekt (akk): ja,

Nominalisierung als Thema des Satzes: -

- Kontext der Nominalisierung:

Hauptsatz: nein, Aussagesatz: - affirmativ: ja,

3. Person: - Singular: - Präsens: -

Indikativ: - Aktiv: -

(3.7) Sie war ganz außer sich vor FREUDE. (185/2)

- zugrundeliegendes Verb des Verbalabstraktums: 
Wortbildung: einfach, Länge: kurz (2 Silben),

Häufigkeit: häufig.

- Verbalabstraktum der Nominalisierung:

Wortbildung: kompliziert, Häufigkeit: häufig,

Bedeutungsvarianten: gleich, Bedeutung: statisch.

- Nominalisierung:

gemeinsame Ergänzungen Verb/Substantiv: sub prp,

Zahl der Ergänzungen der Nominalisierung: 0,

Zahl der Ergänzungen des Verbs minimal: 2 (Aktiv),

Zahl aller Ergänzungen des Verbs: 2 (Aktiv),

Zahl der adnominalen Ergänzungen kleiner: ja,

Subjekt (sub) des Verbs als Attribut: nein,

Direktes Objekt (akk) des V. als Attribut: nein,

Nominalisierung als direktes Objekt (akk): nein,

Nominalisierung als Thema des Satzes: nein.

- Kontext der Nominalisierung:

Hauptsatz: ja, Aussagesatz: ja, affirmativ: ja,

3. Person: ja, Singular: ja, Präsens: nein,

Indikativ: ja, Aktiv: ja.

\section{ZUSTANDSBEZEICHNUNGEN MIT ATTRIBUTIVEN ERGÄNZUNGEN:}

(3.8) Eben noch wollte ich Ihnen meine BEWUNDERUNG ausdrücken. (176/2)

- zugrundeliegendes Verb des Verbalabstraktums:

Wortbildung: kompliziert, Länge: lang (3 Silben),

Häufigkeit: selten.

- Verbalabstraktum der Nominalisierung:

Wortbildung: kompliziert, Häufigkeit: selten,

Bedeutungsvarianten: gleich, Bedeutung: statisch.

- Nominalisierung:

gemeinsame Ergänzungen Verb/Substantiv: sub akk,

Zahl der Ergänzungen der Nominalisierung: 1,

Zahl der Ergänzungen des Verbs minimal: 2 (Aktiv),

Zahl aller Ergänzungen des Verbs: 2 (Aktiv),

Zahl der adnominalen Ergänzungen kleiner: ja,

Subjekt (sub) des Verbs als Attribut: ja (+anim),

Direktes Objekt (akk) des V. als Attribut: nein,

Nominalisierung als direktes Objekt (akk): ja,

Nominalisierung als Thema des Satzes: nein.

- Kontext der Nominalisierung:

Hauptsatz: ja, Aussagesatz: ja, affirmativ: ja, 
3. Person: nein, Singular: ja, Präsens: nein, Indikativ: ja, Aktiv: ja.

(3.9) Sie können erklären, dieses Tier rieche übel, jenes trage einen widerlichen Stachel und wieder jenem fehle das VERMÖGEN zur einfachen Selbsterhaltung. $(181 / 1)$

- zugrundeliegendes Verb des Verbalabstraktums: Wortbildung: kompliziert, Länge: lang (3 Silben), Häufigkeit: häufig.

- Verbalabstraktum der Nominalisierung:

Wortbildung: kompliziert, Häufigkeit: unklar,

Bedeutungsvarianten: gleich, Bedeutung: statisch.

- Nominalisierung:

gemeinsame Ergänzungen Verb/Substantiv: sub akk,

Zahl der Ergänzungen der Nominalisierung: 1,

Zahl der Ergänzungen des Verbs minimal: 2 (Aktiv),

Zahl aller Ergänzungen des Verbs: 2 (Aktiv),

Zahl der adnominalen Ergänzungen kleiner: ja,

Subjekt (sub) des Verbs als Attribut: nein,

Direktes Objekt (akk) des V. als Attribut: ja (-anim),

Nominalisierung als direktes Objekt (akk): nein,

Nominalisierung als Thema des Satzes: nein.

- Kontext der Nominalisierung:

Hauptsatz: nein, Aussagesatz: - affirmativ: ja,

3. Person: ja, Singular: ja, Präsens: ja,

Indikativ: nein, Aktiv: ja.

\section{ERGEBNISBEZEICHNUNGEN OHNE ATTRIBUTIVE ERGÄNZUNGEN:}

(3.10) Ich stelle die AUSNAHME dar. (179/3)

- zugrundeliegendes Verb des Verbalabstraktums:

Wortbildung: kompliziert, Länge: lang (3 Silben),

Häufigkeit: unklar.

- Verbalabstraktum der Nominalisierung:

Wortbildung: kompliziert, Häufigkeit: selten,

Bedeutungsvarianten: weniger, Bedeutung: statisch.

- Nominalisierung:

gemeinsame Ergänzungen Verb/Substantiv: sub akle prp, ${ }^{16}$

16 Die Ergebnisbezeichung unterscheidet sich von der Geschehensbezeichnung meist durch ihre von dem zugrundeliegenden Verb abweichende syntaktische Valenz. Ein Vergleich der Zahl der Ergänzungen von Ergebnisbezeichnungen mit der Zahl der verbalen Ergänzungen erscheint uns 
Zahl der Ergänzungen der Nominalisierung: 0, Zahl der Ergänzungen des Verbs minimal: 2 (W-Passiv)

Zahl aller Ergänzungen des Verbs: 3 (Aktiv),

Zahl der adnominalen Ergänzungen kleiner: -

Subjekt (sub) des Verbs als Attribut: nein,

Direktes Objekt (akk) des V. als Attribut: nein,

Nominalisierung als direktes Objekt (akk): nein,

Nominalisierung als Thema des Satzes: nein.

- Kontext der Nominalisierung:

Hauptsatz: ja, Aussagesatz: ja, affirmativ: ja,

3. Person: nein, Singular: ja, Präsens: ja,

Indikativ: ja, Aktiv: ja.

(3.11) Prüfen wir die ERKLÄRUNGEN, die in Frage kommen. (187/2)

- zugrundeliegendes Verb des Verbalabstraktums:

Wortbildung: kompliziert, Länge: lang (3 Silben),

Häufigkeit: häufig.

- Verbalabstraktum der Nominalisierung:

Wortbildung: kompliziert, Häufigkeit: häufig,

Bedeutungsvarianten: weniger, Bedeutung: statisch.

- Nominalisierung:

gemeinsame Ergänzungen Verb/Substantiv: sub (dat) akk,

Zahl der Ergänzungen der Nominalisierung: 0,

Zahl der Ergänzungen des Verbs minimal: 1 (W-Passiv)

Zahl aller Ergänzungen des Verbs: 3 (Aktiv),

Zahl der adnominalen Ergänzungen kleiner: ja,

Subjekt (sub) des Verbs als Attribut: nein,

Direktes Objekt (akk) des V. als Attribut: nein,

Nominalisierung als direktes Objekt (akk): ja,

Nominalisierung als Thema des Satzes: nein.

- Kontext der Nominalisierung:

Hauptsatz: ja, Aussagesatz: nein, affirmativ: ja,

3. Person: nein, Singular: nein, Präsens: ja,

Indikativ: nein, Aktiv: ja. 


\section{ERGEBNISBEZEICHNUNGEN MIT ATTRIBUTIVEN ERGÄNZUNGEN:}

(3.12) [Die Ehrbegriffe] beruhen auf der VERABREDUNG, daß der Gegner, bei aller Feindschaft, sich seinerseits als ritterlicher Soldat zu bezeigen habe. (172/2)

- zugrundeliegendes Verb des Verbalabstraktums:

Wortbildung: kompliziert, Länge: lang (4 Silben),

Häufigkeit: selten.

- Verbalabstraktum der Nominalisierung:

Wortbildung: kompliziert, Häufigkeit: selten,

Bedeutungsvarianten: mehr, Bedeutung: statisch.

- Nominalisierung:

gemeinsame Ergänzungen Verb/Substantiv: sub akk prp,

Zahl der Ergänzungen der Nominalisierung: 1,

Zahl der Ergänzungen des Verbs minimal: 1 (W-Passiv)

Zahl aller Ergänzungen des Verbs: 3 (Aktiv),

Zahl der adnominalen Ergänzungen kleiner: nein,

Subjekt (sub) des Verbs als Attribut: nein,

Direktes Objekt (akk) des V. als Attribut: ja (-anim),

Nominalisierung als direktes Objekt (akk): nein,

Nominalisierung als Thema des Satzes: nein.

- Kontext der Nominalisierung:

Hauptsatz: ja, Aussagesatz: ja, affirmativ: ja,

3. Person: ja, Singular: nein, Präsens: ja,

Indikativ: ja, Aktiv: ja.

(3.13) Seine VERBRECHEN sind ungeheuerlich, ... (180/5)

- zugrundeliegendes Verb des Verbalabstraktums:

Wortbildung: kompliziert, Länge: lang (3 Silben),

Häufigkeit: selten.

- Verbalabstraktum der Nominalisierung:

Wortbildung: kompliziert, Häufigkeit: selten,

Bedeutungsvarianten: weniger, Bedeutung: statisch.

- Nominalisierung:

gemeinsame Ergänzungen Verb/Substantiv: sub akk,

Zahl der Ergänzungen der Nominalisierung: 1,

Zahl der Ergänzungen des Verbs minimal: 2 (Aktiv)

Zahl aller Ergänzungen des Verbs: 2 (Aktiv),

Zahl der adnominalen Ergänzungen kleiner: -

Subjekt (sub) des Verbs als Attribut: ja (+anim),

Direktes Objekt (akk) des V. als Attribut: nein,

Nominalisierung als direktes Objekt (akk): nein,

Nominalisierung als Thema des Satzes: ja. 
- Kontext der Nominalisierung:

Hauptsatz: ja, Aussagesatz: ja, affirmativ: ja,

3. Person: ja, Singular: nein, Präsens: ja,

Indikativ: ja, Aktiv: ja.

FUNKTIONSVERBGEFÜGE (FUNKTIONSNOMEN OHNE ATTRIBUTIVE ERGÄNZUNGEN): ${ }^{17}$

(3.14) Das flüssige Gestein erstarrt, wo es mit der Luft in BERÜHRUNG kommt. (192/3)

- zugrundeliegendes Verb des Verbalabstraktums:

Wortbildung: kompliziert, Länge: lang (3 Silben),

Häufigkeit: selten.

- Verbalabstraktum der Nominalisierung:

Wortbildung: kompliziert, Häufigkeit: selten,

Bedeutungsvarianten: gleich, Bedeutung: dynamisch.

- Nominalisierung:

gemeinsame Ergänzungen Verb/Substantiv: sub akk,

Zahl der Ergänzungen der Nominalisierung: 2,

Zahl der Ergänzungen des Verbs minimal: 2 (Aktiv)

Zahl aller Ergänzungen des Verbs: 2 (Aktiv),

Zahl der adnominalen Ergänzungen kleiner: -

Subjekt (sub) des Verbs als Attribut: nein,

Direktes Objekt (akk) des V. als Attribut: nein,

Nominalisierung als direktes Objekt (akk): nein,

Nominalisierung als Thema des Satzes: nein.

- Kontext der Nominalisierung:

Hauptsatz: nein, Aussagesatz: - affirmativ: ja,

3. Person: ja, Singular: ja, Präsens: ja, Indikativ: ja, Aktiv: ja.

(3.15) Ihre Worte setzen mich in ERSTAUNEN. (213/3)

- zugrundeliegendes Verb des Verbalabstraktums:

Wortbildung: kompliziert, Länge: lang (3 Silben),

Häufigkeit: selten.

- Verbalabstraktum der Nominalisierung:

Wortbildung: kompliziert, Häufigkeit: selten,

Bedeutungsvarianten: weniger, Bedeutung: statisch.

- Nominalisierung:

17 Als Attribut von Funktionsverbgefügen gelten in dieser Untersuchung nur nicht frei im Satz verschiebbare Ergänzungen (vgl. Helbig/Buscha 1987:102). 
gemeinsame Ergänzungen Verb/Substantiv: sub akk,

Zahl der Ergänzungen der Nominalisierung: 2,

Zahl der Ergänzungen des Verbs minimal: 2 (Aktiv)

Zahl aller Ergänzungen des Verbs: 3 (Aktiv),

Zahl der adnominalen Ergänzungen kleiner: -

Subjekt (sub) des Verbs als Attribut: nein,

Direktes Objekt (akk) des V. als Attribut: nein,

Nominalisierung als direktes Objekt (akk): nein,

Nominalisierung als Thema des Satzes: nein.

- Kontext der Nominalisierung:

Hauptsatz: ja, Aussagesatz: ja, affirmativ: ja,

3. Person: ja, Singular: nein, Präsens: ja,

Indikativ: ja, Aktiv: ja.

(3.16) Wissen Sie, Professor Simon, ich habe VERSTÄNDNIS für ihn. (210/5)

- zugrundeliegendes Verb des Verbalabstraktums:

Wortbildung: kompliziert, Länge: lang (3 Silben),

Häufigkeit: häufig.

- Verbalabstraktum der Nominalisierung:

Wortbildung: kompliziert, Häufigkeit: selten,

Bedeutungsvarianten: weniger, Bedeutung: statisch.

- Nominalisierung:

gemeinsame Ergänzungen Verb/Substantiv: sub akk,

Zahl der Ergänzungen der Nominalisierung: 2,

Zahl der Ergänzungen des Verbs minimal: 2 (Aktiv)

Zahl aller Ergänzungen des Verbs: 2 (Aktiv),

Zahl der adnominalen Ergänzungen kleiner: -

Subjekt (sub) des Verbs als Attribut: nein,

Direktes Objekt (akk) des V. als Attribut: nein,

Nominalisierung als direktes Objekt (akk): nein, ${ }^{18}$

Nominalisierung als Thema des Satzes: nein.

- Kontext der Nominalisierung:

Hauptsatz: nein, Aussagesatz: - affirmativ: ja,

3. Person: nein, Singular: ja, Präsens: ja,

Indikativ: ja, Aktiv: ja. 
(3.17) Übrigens, ich habe die ENTDECKUNG gemacht, daß Verdi und Offenbach ein und derselbe Komponist sind. (216/4)

- zugrundeliegendes Verb des Verbalabstraktums:

Wortbildung: kompliziert, Länge: lang (3 Silben),

Häufigkeit: selten.

- Verbalabstraktum der Nominalisierung:

Wortbildung: kompliziert, Häufigkeit: selten,

Bedeutungsvarianten: weniger, Bedeutung: statisch.

- Nominalisierung:

gemeinsame Ergänzungen Verb/Substantiv: sub akk,

Zahl der Ergänzungen der Nominalisierung: 2 (1 attributive)

Zahl der Ergänzungen des Verbs minimal: 2 (Aktiv)

Zahl aller Ergänzungen des Verbs: 2 (Aktiv),

Zahl der adnominalen Ergänzungen kleiner: -

Subjekt (sub) des Verbs als Attribut: nein,

Direktes Objekt (akk) des V. als Attribut: ja (-anim),

Nominalisierung als direktes Objekt (akk): nein,

Nominalisierung als Thema des Satzes: nein.

- Kontext der Nominalisierung:

Hauptsatz: ja, Aussagesatz: ja, affirmativ: ja,

3. Person: nein, Singular: ja, Präsens: nein,

Indikativ: ja, Aktiv: ja.

(3.18) Im April dieses Jahres faßten wir den BESCHLUSS der Mexikanisierung des

Krieges. $(212 / 2)$

- zugrundeliegendes Verb des Verbalabstraktums:

Wortbildung: kompliziert, Länge: lang (3 Silben),

Häufigkeit: selten.

- Verbalabstraktum der Nominalisierung:

Wortbildung: einfach, Häufigkeit: selten,

Bedeutungsvarianten: weniger, Bedeutung: statisch.

- Nominalisierung:

gemeinsame Ergänzungen Verb/Substantiv: sub akk,

Zahl der Ergänzungen der Nominalisierung: 2 (1 attributive)

Zahl der Ergänzungen des Verbs minimal: 2 (Aktiv)

Zahl aller Ergänzungen des Verbs: 2 (Aktiv),

Zahl der adnominalen Ergänzungen kleiner: -

Subjekt (sub) des Verbs als Attribut: nein,

Direktes Objekt (akk) des V. als Attribut: ja (-anim), 
Nominalisierung als direktes Objekt (akk): nein,

Nominalisierung als Thema des Satzes: nein.

- Kontext der Nominalisierung:

Hauptsatz: ja, Aussagesatz: ja, affirmativ: ja,

3. Person: nein, Singular: nein, Präsens: nein,

Indikativ: ja, Aktiv: ja.

(3.19) Herr, Sie stehen unter meinem BEFEHL. (178/1)

- zugrundeliegendes Verb des Verbalabstraktums:

Wortbildung: kompliziert, Länge: lang (3 Silben),

Häufigkeit: selten.

- Verbalabstraktum der Nominalisierung:

Wortbildung: einfach, Häufigkeit: häufig,

Bedeutungsvarianten: weniger, Bedeutung: statisch.

- Nominalisierung:.

gemeinsame Ergänzungen Verb/Substantiv: sub dat akk,

Zahl der Ergänzungen der Nominalisierung: 2 (1 attributive)

Zahl der Ergänzungen des Verbs minimal: 3 (Aktiv)

Zahl aller Ergänzungen des Verbs: 3 (Aktiv),

Zahl der adnominalen Ergänzungen kleiner: -

Subjekt (sub) des Verbs als Attribut: ja (tanim),

Direktes Objekt (akk) des V. als Attribut: nein,

Nominalisierung als direktes Objekt (akk): nein,

Nominalisierung als Thema des Satzes: nein.

- Kontext der Nominalisierung:

Hauptsatz: ja, Aussagesatz: ja, affirmativ: ja,

3. Person: ja, Singular: nein, Präsens: ja,

Indikativ: ja, Aktiv: ja.

\section{BEISPIELE AUS DEM GRUNDSAMPLE 'B'}

GESCHEHENSBEZEICHNUNGEN OHNE ATTRIBUTIVE ERGÄNZUNGEN:

(3.20) Und man soll nicht immer gleich nach der Regierung oder

REGLEMENTIERUNG schreien. (Verkehrssicherheit 88/1)

- zugrundeliegendes Verb des Verbalabstraktums:

Wortbildung: kompliziert, Länge: lang (5 Silben),

Häufigkeit: selten.

- Verbalabstraktum der Nominalisierung:

Wortbildung: kompliziert, Häufigkeit: selten,

Bedeutungsvarianten: gleich, Bedeutung: dynamisch.

- Nominalisierung: 
gemeinsame Ergänzungen Verb/Substantiv: sub akk,

Zahl der Ergänzungen der Nominalisierung: 0,

Zahl der Ergänzungen des Verbs minimal: 1 (W-Passiv)

Zahl aller Ergänzungen des Verbs: 2 (Aktiv),

Zahl der adnominalen Ergänzungen kleiner: ja,

Subjekt (sub) des Verbs als Attribut: nein,

Direktes Objekt (akk) des V. als Attribut: nein,

Nominalisierung als direktes Objekt (akk): nein,

Nominalisierung als Thema des Satzes: nein.

- Kontext der Nominalisierung:

Hauptsatz: ja, Aussagesatz: ja, affirmativ: nein,

3. Person: ja, Singular: ja, Präsens: ja,

Indikativ: ja, Aktiv: ja.

(3.21) Zum Beispiel werden doch viele Unfälle durch ÜBERHOLEN in unübersichtlichen Kurven verursacht. (Verkehrssicherheit 105/1)

- zugrundeliegendes Verb des Verbalabstraktums:

Wortbildung: kompliziert, Länge: lang (4 Silben),

Häufigkeit: selten.

- Verbalabstraktum der Nominalisierung:

Wortbildung: kompliziert, Häufigkeit: selten,

Bedeutungsvarianten: unklar, Bedeutung: dynamisch.

- Nominalisierung:

gemeinsame Ergänzungen Verb/Substantiv: sub (akk),

Zahl der Ergänzungen der Nominalisierung: 0,

Zahl der Ergänzungen des Verbs minimal: 0 (W-Passiv)

Zahl aller Ergänzungen des Verbs: 2 (Aktiv),

Zahl der adnominalen Ergänzungen kleiner: nein,

Subjekt (sub) des Verbs als Attribut: nein,

Direktes Objekt (akk) des V. als Attribut: nein,

Nominalisierung als direktes Objekt (akk): nein,

Nominalisierung als Thema des Satzes: nein.

- Kontext der Nominalisierung:

Hauptsatz: ja, Aussagesatz: ja, affirmativ: ja,

3. Person: ja, Singular: nein, Präsens: ja,

Indikativ: ja, Aktiv: nein.

(3.22) Aber das ZEICHNEN nebenbei und zwischendurch das läßt sich ohne weiteres einbauen, ... (Grass 164/3)

- zugrundeliegendes Verb des Verbalabstraktums:

Wortbildung: einfach, Länge: lang (2 Silben),

Häufigkeit: häufig. 
- Verbalabstraktum der Nominalisierung:

Wortbildung: kompliziert, Häufigkeit: selten,

Bedeutungsvarianten: unklar, Bedeutung: dynamisch.

- Nominalisierung:

gemeinsame Ergänzungen Verb/Substantiv: sub (akk),

Zahl der Ergänzungen der Nominalisierung: 0,

Zahl der Ergänzungen des Verbs minimal: 1 (Aktiv),

Zahl aller Ergänzungen des Verbs: 2 (Aktiv),

Zahl der adnominalen Ergänzungen kleiner: ja,

Subjekt (sub) des Verbs als Attribut: nein,

Direktes Objekt (akk) des V. als Attribut: nein,

Nominalisierung als direktes Objekt (akk): nein,

Nominalisierung als Thema des Satzes: ja.

- Kontext der Nominalisierung:

Hauptsatz: ja, Aussagesatz: ja, affirmativ: ja,

3. Person: ja, Singular: ja, Präsens: ja,

Indikativ: ja, Aktiv: ja.

\section{GESCHEHENSBEZEICHNUNGEN MIT ATTRIBUTIVEN ERGÄNZUNGEN:}

(3.23) Erst einmal möchte ich sagen, daß ich bedaure, daß ich die einzige der Frauen hier bin, die für die ABSCHAFFUNG des Paragraphen eintreten.

(Abtreibung 368/1)

- zugrundeliegendes Verb des Verbalabstraktums:

Wortbildung: kompliziert, Länge: lang (3 Silben),

Häufigkeit: selten.

- Verbalabstraktum der Nominalisierung:

Wortbildung: kompliziert, Häufigkeit: selten,

Bedeutungsvarianten: weniger, Bedeutung: dynamisch.

- Nominalisierung:

gemeinsame Ergänzungen Verb/Substantiv: sub akk,

Zahl der Ergänzungen der Nominalisierung: 1,

Zahl der Ergänzungen des Verbs minimal: 1 (W-Passiv)

Zahl aller Ergänzungen des Verbs: 2 (Aktiv),

Zahl der adnominalen Ergänzungen kleiner: nein,

Subjekt (sub) des Verbs als Attribut: nein,

Direktes Objekt (akk) des V. als Attribut: ja (-anim),

Nominalisierung als direktes Objekt (akk): nein,

Nominalisierung als Thema des Satzes: nein.

- Kontext der Nominalisierung:

Hauptsatz: nein, Aussagesatz: - affirmativ: ja, 
3. Person: ja, Singular: nein, Präsens: ja, Indikativ: ja, Aktiv: ja.

(3.24) Aber ich glaube, jede SPEKULATION auf das Publikum also direkt gezielt ist nicht etwa moralisch verwerflich. (Grass 155/2)

- zugrundeliegendes Verb des Verbalabstraktums:

Wortbildung: kompliziert, Länge: lang (4 Silben), Häufigkeit: selten.

- Verbalabstraktum der Nominalisierung:

Wortbildung: kompliziert, Häufigkeit: selten, Bedeutungsvarianten: weniger, Bedeutung: dynamisch.

- Nominalisierung:

gemeinsame Ergänzungen Verb/Substantiv: sub prp,

Zahl der Ergänzungen der Nominalisierung: 1,

Zahl der Ergänzungen des Verbs minimal: 1 (Infin.)

Zahl aller Ergänzungen des Verbs: 2 (Aktiv),

Zahl der adnominalen Ergänzungen kleiner: nein,

Subjekt (sub) des Verbs als Attribut: nein,

Direktes Objekt (akk) des V. als Attribut: nein,

Nominalisierung als direktes Objekt (akk): nein,

Nominalisierung als Thema des Satzes: ja.

- Kontext der Nominalisierung:

Hauptsatz: nein, Aussagesatz: - affirmativ: nein,

3. Person: ja, Singular: ja, Präsens: ja,

Indikativ: ja, Aktiv: ja.

(3.25) Die VERFÜHRUNG zum Trinken ist natürlich zum Teil sogar offiziell sanktioniert, ... (Verkehrssicherheit 113/1)

- zugrundeliegendes Verb des Verbalabstraktums:

Wortbildung: kompliziert, Länge: lang (3 Silben),

Häufigkeit: selten.

- Verbalabstraktum der Nominalisierung:

Wortbildung: kompliziert, Häufigkeit: selten,

Bedeutungsvarianten: mehr, Bedeutung: dynamisch.

- Nominalisierung:

gemeinsame Ergänzungen Verb/Substantiv: sub akk prp,

Zahl der Ergänzungen der Nominalisierung: 1,

Zahl der Ergänzungen des Verbs minimal: 2 (W-Passiv)

Zahl aller Ergänzungen des Verbs: 3 (Aktiv),

Zahl der adnominalen Ergänzungen kleiner: ja,

Subjekt (sub) des Verbs als Attribut: nein,

Direktes Objekt (akk) des V. als Attribut: nein, 
Nominalisierung als direktes Objekt (akk): nein,

Nominalisierung als Thema des Satzes: ja.

- Kontext der Nominalisierung:

Hauptsatz: ja, Aussagesatz: ja, affirmativ: ja,

3. Person: ja, Singular: ja, Präsens: ja,

Indikativ: ja, Aktiv: nein.

\section{ZUSTANDSBEZEICHNUNGEN OHNE ATTRIBUTIVE ERGÄNZUNGEN:}

(3.26) Wir versuchen, die beim Fang auftretende ERREGUNG auf den kleinsten Grad herabzusetzen. (Fische 191/4)

- zugrundeliegendes Verb des Verbalabstraktums:

Wortbildung: kompliziert, Länge: lang (3 Silben),

Häufigkeit: selten.

- Verbalabstraktum der Nominalisierung:

Wortbildung: kompliziert, Häufigkeit: selten,

Bedeutungsvarianten: weniger, Bedeutung: statisch.

- Nominalisierung:

gemeinsame Ergänzungen Verb/Substantiv: sub <akk,

Zahl der Ergänzungen der Nominalisierung: 0,

Zahl der Ergänzungen des Verbs minimal: 1 (S-Passiv),

Zahl aller Ergänzungen des Verbs: 2 (Aktiv),

Zahl der adnominalen Ergänzungen kleiner: ja,

Subjekt (sub) des Verbs als Attribut: nein,

Direktes Objekt (akk) des V. als Attribut: nein,

Nominalisierung als direktes Objekt (akk): ja,

Nominalisierung als Thema des Satzes: -

- Kontext der Nominalisierung:

Hauptsatz: nein, Aussagesatz: - affirmativ: ja,

3. Person: - Singular: - Präsens: -

Indikativ: - Aktiv: -

(3.27) Aber eine gewisse RESIGNATION hat sich doch ausgebreitet.

(Verkehrssicherheit 71/1)

zugrundeliegendes Verb des Verbalabstraktums:

Wortbildung: kompliziert, Länge: lang (4 Silben),

Häufigkeit: selten.

- Verbalabstraktum der Nominalisierung:

Wortbildung: kompliziert, Häufigkeit: selten,

Bedeutungsvarianten: mehr, Bedeutung: statisch.

- Nominalisierung:

gemeinsame Ergänzungen Verb/Substantiv: sub, 
Zahl der Ergänzungen der Nominalisierung: 0,

Zahl der Ergänzungen des Verbs minimal: 1 (Aktiv),

Zahl aller Ergänzungen des Verbs: 1 (Aktiv),

Zahl der adnominalen Ergänzungen kleiner: ja,

Subjekt (sub) des Verbs als Attribut: nein,

Direktes Objekt (akk) des V. als Attribut: nein,

Nominalisierung als direktes Objekt (akk): nein,

Nominalisierung als Thema des Satzes: ja.

- Kontext der Nominalisierung:

Hauptsatz: ja, Aussagesatz: ja, affirmativ: ja,

3. Person: ja, Singular: ja, Präsens: nein,

Indikativ: ja, Aktiv: ja.

\section{ZUSTANDSBEZEICHNUNGEN MIT ATTRIBUTIVEN ERGÄNZUNGEN:}

(3.28) Wie stehen Sie zu der Tatsache, daß ... Ärzte dafür stimmten, daß eine schwere SCHÄDIGUNG des Embrvos den Abbruch der Schwangerschaft rechtfertigen kann? (Abtreibung 374/2)

- zugrundeliegendes Verb des Verbalabstraktums:

Wortbildung: kompliziert, Länge: lang (3 Silben),

Häufigkeit: selten.

- Verbalabstraktum der Nominalisierung:

Wortbildung: kompliziert, Häufigkeit: selten,

Bedeutungsvarianten: mehr, Bedeutung: statisch.

- Nominalisierung:

gemeinsame Ergänzungen Verb/Substantiv: sub $<<$ akk (prp),

Zahl der Ergänzungen der Nominalisierung: 1,

Zahl der Ergänzungen des Verbs minimal: 1 (S-Passiv),

Zahl aller Ergänzungen des Verbs: 3 (Aktiv),

Zahl der adnominalen Ergänzungen kleiner: nein,

Subjekt (sub) des Verbs als Attribut: ja (+anim), ${ }^{19}$

Direktes Objekt (akk) des V. als Attribut: nein,

Nominalisierung als direktes Objekt (akk): nein,

Nominalisierung als Thema des Satzes: ja.

- Kontext der Nominalisierung:

Hauptsatz: nein, Aussagesatz: - affirmativ: ja,

3. Person: ja, Singular: ja, Präsens: ja,

Indikativ: ja, Aktiv: ja. 
(3.29) Aber wie haben wir es dann mit der UNFALLBETEILIGUNG der Jugendlichen bis 25 Jahren, Herr Dr. Seehum ? (Verkehrssicherheit 124/5)

- zugrundeliegendes Verb des Verbalabstraktums:

Wortbildung: kompliziert, Länge: lang (4 Silben),

Häufigkeit: selten.

- Verbalabstraktum der Nominalisierung:

Wortbildung: kompliziert, Häufigkeit: selten,

Bedeutungsvarianten: weniger, Bedeutung: statisch.

- Nominalisierung:

gemeinsame Ergänzungen Verb/Substantiv: sub prp,

Zahl der Ergänzungen der Nominalisierung: 1,

Zahl der Ergänzungen des Verbs minimal: 2 (S-Passiv),

Zahl aller Ergänzungen des Verbs: 2 (Aktiv),

Zahl der adnominalen Ergänzungen kleiner: ja,

Subjekt (sub) des Verbs als Attribut: ja (+anim),

Direktes Objekt (akk) des V. als Attribut: nein,

Nominalisierung als direktes Objekt (akk): nein,

Nominalisierung als Thema des Satzes: nein.

- Kontext der Nominalisierung:

Hauptsatz: ja, Aussagesatz: nein, affirmativ: ja,

3. Person: nein, Singular: nein, Präsens: ja,

Indikativ: ja, Aktiv: ja.

In den folgenden beiden Listen werden die einzelnen Verbalabstrakta und ihre in den Grundsamples auftretende Vorkommenshäufigkeit (insofern sie größer als 1 ist, folgt die entsprechende Zahl dem Verbalabstraktum) aufgeführt (vgl. auch Tabelle 3, die Tortendiagramme 3, 4 und Diagramm 5).

Grundsample A (geordnet nach Ableitungsaffix und Alphabet)

[-0]: Ablauf, Angriff, Anschlag, Anspruch, Antwort, Arbeit, Auftrag 4, Ausdruck, Ausschau, Befehl 4, Begriff, Beistand, Bericht, Beschluß, Besitz 2, Blick 3, Durchbruch, Einfall, Empfang, Entschluß 2, Fang, Fehltritt, Flirt, Fortschritt, Furcht, Geduld 3, Geleitschutz, Genuß, Hohn, Kuß, Morgenspaziergang, Nachweis, Rat 2, Schluß 2, Schuß, Selbstvorwurf 2, Sieg, Spiel 3, Sprung, Stand, Sternenumlauf, Stoß, Todesschreck, Trost, Truppenabzug, Untergang, Unterschied 2, Urteil, Verdacht, Verfolg, Vorgang 3, Vortrag 3, Widerspruch 2, Widerstand 2, Wunsch, Zahnwechsel, Zusammenarbeit, Zusammenbruch, Zusammenhang, Zutritt, Zweikampf, Zwergenwuchs;

[-e]: Absprache, Annahme, Aufgabe 4, Ausnahme 3, Aussprache, Folge 2, Forschungsreise, Frage 2, Hilfe, Liebe 2, Revanche, Sorge; 
[-en]: Abendessen, Abwerfen, Austrocknen, Dasein, Entsetzen, Erstaunen, Fischen 2, Gehen, Leben 8, Mikroskopieren, Stillschweigen, Unternehmen, Verbrechen 3, Verfahren 2, Vergnügen 2, Verhalten 4, Vermögen, Vertrauen 2, Vorhaben, Vorkommen;

[-(er)ei]: Schlamperei, Spionenriecherei;

[Ge- (-e)]: Gedanke, Gefühl, Geschrei 2, Geschwätz, Gespräch;

[-nis]: Bedürfnis, Befugnis, Ergebnis, Geständnis 2, Kenntnis, Verständnis 2, Vorkommnis;

[-ung]: Abweichung, Anordnung 2, Anstrengung, Anweisung 3, Aufklärung 2, Ausfallerscheinung, Ausführung, Ausübung, Bedeutung 2, Behauptung 2, Belagerung, Bemerkung 2, Benennung, Beratung, Berührung, Betonung, Beweisführung 2, Bewunderung, Einhaltung, Entdeckung 3, Entgegnung, Entschuldigung, Entwicklung, Erhaltung, Erklärung 11, Erregung, Erschütterung, Handlung 2, Hoffnung 2, Kriegsführung, Leichenöffnung, Leistung 2, Leitung, Meinung 3, Messung, Mutmaßung 2, Ordnung 2, Regung, Reinhaltung, Schädelmessung, Scheidung, Selbstachtung, Störung, Umwandlung, Unterredung, Unterstützung, Überlegung, Überraschung 2, Überschwemmung 2, Überzeugung, Verabredung, Veränderung, Verfolgung, Verfügung, Verteidigung 2, Verwandlung 2, Verwechslung, Verwirrung, Vorbereitung, Wahrnehmung 2, Wasseratmung, Wechselwirkung 3, Weisung, Zusicherung, Zustimmung;

[-(s)t/d(e)]: Beschwerde 2, Einsicht, Freude, Rückfahrt 2, Rücksicht, Tat, Verlust 2;

[-tum]: Irrtum 2, Wachstum;

[-(at)ion]: Operation.

Grundsample B (geordnet nach Ableitungsaffix und Alphabet)

[-0]: Abbruch 2, Ablauf, Abtreibungszwang, Anfang, Arbeit 6, Ausschau, Beginn 3, Bezug, Blick, Detektivarbeit, Fang, Ferienbeginn, Flirt, Furcht, Geduld, Genuß, Kauf, Kritik, Lesearbeit, Morgenspaziergang, Nachweis, Protest, Schuß, Schutz 4, Schwangerschaftsabbruch 2, Sieg, Sprung, Truppenabzug, Unfallflucht 2, Urteil 3, Überholbeginn, Vergleich, Zahnwechsel, Zusammenarbeit, Zutritt, Zweikampf;

[-e]: Anfrage, Auslese, Aussprache, Bestandaufnahme, Gegenauslese, Interesse 3, Reise, Revanche, Rücksichtsnahme, Umfrage 2;

[-en]: Abwerfen, Ankreuzen 2, Ausmorden, Austrocknen, Autofahren 3, Erreichen, Fahren 6, Fischen 2, Führen 2, Jugendfreie-Texte-und-nicht-jugendfreie-Texte-Unterscheiden, Linksüberholen, Mikroskopieren, Nichtwissen, Nicken, Rechtsüberholen, Reduzieren, Romanschreiben, Schreiben, Tabu-Einreißen, Töten, Trinken, Unterhalten, Überholen 3, Zeichnen;

[-(er)ei]: Gerüchtemacherei, Quälerei 2, Schlamperei, Spielerei, Spionenriecherei, Tierquälerei; 
[Ge- (-e)]: Geschrei 2, Geschwätz;

[-ung]: Abdunklung, Abschaffung, Abtreibung 5, Aburteilung, Anpassung 2, Anschaffung, Anstrengung, Aufklärung 3, Auftragverwaltung, Ausbildung, Ausführung, Ausübung, Änderung 4, Befreiung, Befruchtung 2, Begünstigung, Belagerung, Benachteiligung, Benennung, Berufsausbildung, Beschreibung 4, Besserung, Bestellung, Bestrafung, Betonung, Bewältigung, Beweisführung 2, Bildung, Diffamierung, Einführung, Einhaltung, Einnistung 4, Einpassung, Einschränkung, Entdeckung 2, Entwicklung, Erhaltung, Erklärung, Erläuterung, Erneuerung, Erregung, Erziehung 2, Formulierung 2, Geheimhaltung, Geschwindigkeitsbegrenzung 2, Geschwindigkeitsbeschränkung, Geschwindigkeitsüberschreitung, Gewinnbeteiligung, Güteabwägung, Handhabung, Hilfeleistung, Intelligenzprüfung, Klärung, Koordinierung 5, Kriegsführung, Leitung, Lockerung, Lösung, Messung, Prüfung 12, Rechtssprechung, Regelung 2, Reglementierung, Regung, Reinhaltung, Rote-Kreuz-Stationierung, Satzverkürzung, Schädelmessung, Schädigung, Scheidung, Selbstachtung, Selbstbestimmung, Störung, Strafandrohung, Strafzumessung, Streichung, Substantivierung, Tötung 2, Umkehrung, Umwandlung, Unfallbeteiligung, Unfallmeldung, Unfallverhütung, Unterhaltung, Unterredung, Unterstützung, Untersuchung, Überlegung, Überschreitung, Überschwemmung 2, Verbesserung, Verbindung, Verfolgung, Verführung, Verkürzung, Vernichtung, Verringerung, Verteidigung 2, Verwandlung, Verwechslung, Verwirrung, Vorbereitung 2, Wasseratmung, Wechselwirkung 3;

[-(s)t/d(e)]: Abfahrt, Freude, Rückfahrt 2, Verlust;

[-tum]: Irrtum, Wachstum;

[-(at)ion]: Diskussion, Emanzipation, Konsultation, Operation, Resignation, Spekulation 3, Variation.

\section{DIE STATISTISCHEN ERGEBNISSE}

Die Vorhersagen 2.12.20 wurden anhand eines Korpus geprüft. Als Korpus diente Das Theaterstück "Die Fische" von Peter Hacks und die Diskussionen "Fragen der Verkehrssicherheit", "Schulklassengespräch mit Günter Grass" sowie "Moral 71. Zum Beispiel Abtreibung". Aus diesem Material wurden vier Stichproben gebildet: zwei Grundsamples (Grundsample A und Grundsample B) und zwei Kontrollsamples (Kontrollsample A und Kontrollsample B).

Das Grundsample A, gebildet auf der Grundlage des Theaterstücks "Die Fische" von Peter Hacks, enthält 256 Sätze und satzwertige Konstruktionen, die Nominalisierungen mit Satzgliedcharakter enthalten. Das Grundsample A wurde mit dem Kontrollsample A verglichen. Das Kontrollsample A enthält 1212 Sätze und satzwertige Konstruktionen ohne Nominalisierungen mit Satzgliedcharakter. 
Die Sätze der beiden Stichproben sind nach bestimmten Kriterien klassifiziert und statistisch ausgewertet worden. Die Ergebnisse lassen sich folgendermaßen tabellarisch zusammenfassen:

\section{TABELLE 1: VERGLEICH GRUNDSAMPLE A MIT KONTROLLSAMPLE $A^{20}$}

$\begin{array}{lccc}\text { DIE ENTSPRECHENDE } & \text { GRUNDSAMPLE } & \text { KONTROLLSAMPLE } & \mathrm{I}_{\mathrm{d}} \\ \text { VORHERSAGE } & \text { Werte } \% & \text { Werte } \% & \end{array}$

\section{A1. ZUGRUNDELIEGENDES VERB (GS) : SATZVERB (KS)}

1. kompliziert gebautes Verb einfach gebautes Verb Samplegröße

2. langes Verb $<36$ Silben $>$ kurzes Verb $<12$ Silben $>$ Samplegröße

3. seltenes Verb häufiges Verb + unklar Samplegröße

$\begin{array}{rr}184 & 66.91 \\ 91 & 33.09 \\ 275 & 100.00 \\ 184 & 66.91 \\ 91 & 33.09 \\ 275 & 100.00 \\ 117 & 42.55 \\ 158 & 57.45 \\ 275 & 100.00\end{array}$

464 683 40.45 59.55 1147 100.00

$\begin{array}{rrr}469 & 40.89 & \pm 7.78 \\ 678 & 59.11 & \\ 1147 & 100.00 & \\ 340 & 29.64 & \pm 4.11 \\ 807 & 70.36 & \\ 1147 & 100.00 & \end{array}$

A2. KERN DER NOMINALISIERUNG (GS): KERN DER SUBSTANTIVGRUPPE (KS)

4. kompliziert gebautes Subst. 199 72.36 469 45.45 $+7.93$ einfach gebautes Substantiv

$76 \quad 27.64$
563 54.55 Samplegröße

$275 \quad 100.00$

1032

100.00

5. selten vorkommendes Subst.

191

69.45

$84 \quad 30.55$

558

54.07

häufiges Subst. + unklar

275

100.00

474

45.93

Samplegröße

170

$$
61.82
$$

1032

100.00

6. bedeutungsenger als Verb nicht bedeutungsenger als $\mathrm{V}$

105

38.18

137.5

50.00

$+2.79$ Samplegröße

$275 \quad 100.00$

137.5

50.00

$275.0 \quad 100.00$

B1. NOMINALISIERUNG (GS) : SUBSTANTTVGRUPPE / BASISVERBPHRASE (KS)

7. weniger Ergänzungen $<0>$ $91 \quad 55.83$ 8 $4.91+10.00$ als Verbalphrase im Satz

20 Der Differenzindex $\left(I_{d}\right)$ wird nach folgender Formel berechnet (Pavlić 1971, 226-229): $I_{d}=\left(P_{1}\right.$ $\left.P_{2}\right) / s_{d}$, wobei $P_{1}$ und $P_{2}$ die beiden aus den Samples ermittelten Werte eines grammatischen Parameters sind und sich $s_{d}$ aus folgender Formel berechnen läßt $s_{d}=\left(p^{*} q^{*}\left(n_{1}+n_{2}\right) /\left(n_{1}+\right.\right.$ $\mathrm{n} 2))^{1 / 2}$. Der Unterschied zwischen Grund-und Kontrollsample ist statistisch signifikant, wenn der Wert von $I_{d}>2$ ist.

In Tabelle 1 und 2 sind Indexwerte, die signifikante Unterschiede anzeigen, unterstrichen. Die positiven Indexwerte in Tabelle 1 und 2 sowie in Diagramm 1 und 2 bestätigen unsere Vorhersagen bzw. zeigen die Tendenz zur Bestätigung unserer Vorhersagen, während die negativen Indexwerte in Tabelle 1 und 2 sowie in Diagramm 1 und 2 unsere Vorhersagen verwerfen bzw. die Tendenz zur Verwerfung unserer Vorhersagen zeigen. 
mehr Ergänzungen <14>

als Verbalphrase im Satz

Teilsamplegröße

Funktionsverbgefüge

Valenzvergleich unmöglich

pronominalisierte Subst.

Samplegröße

8. Subjektgenitiv belebt

Subjektgenitiv unbelebt

Samplegröße

9. Objektgenitiv unbelebt

Objektgenitiv belebt

Samplegröße

10 Nom. als direktes Objekt

Nom. als anderes Satzglied

Samplegröße

11 NP als Thema des Satzes

NP als Rhema des Satzes

Samplegröße
72

44.17

$163 \quad 100.00$

54

39

19

275

$\begin{array}{rrrrr}64 & 95.52 & 694 & 71.03 & \underline{+4.35} \\ 3 & 4.48 & 283 & 28.97 & \\ 67 & 100.00 & 977 & 100.00 & \\ 23 & 82.14 & 196 & 61.83 & \underline{+2.14} \\ 5 & 17.86 & 121 & 38.17 & \\ 28 & 100.00 & 317 & 100.00 & \\ 70 & 25.45 & 317 & 15.91 & \pm 3.94 \\ 205 & 74.55 & 1675 & 84.09 & \\ 275 & 100.00 & 1992 & 100.00 & \\ 43 & 18.30 & 239 & 28.66 & \underline{-3.18} \\ 192 & 81.70 & 595 & 71.34 & \\ 235 & 100.00 & 834 & 100.00 & \end{array}$

B2. KONTEXT DER NOMINALISIERUNG : KONTEXT DER SUBSTANTIVGRUPPE

12 häufiger in Hauptsätzen

seltener in Nebensätzen

Teilsamplegröße

Infinitivsätze

Partizipialphrasen

Samplegröße

13 in NS mit infinitem Verb

in NS mit finitem Verb

Samplegröße

14 häufiger in Aussagesätzen

seltener in Nicht-Aussages.

Samplegröße

15 häufiger in bejahenden

seltener in verneinenden $\mathrm{S}$.

Samplegröße

16 Verb in dritter Person

Verb in nichtdritter Pers.

Samplegröße

17 Verb im Singular
179

56

235

19

2

256

21

56

77

155

24

179

$\mathrm{S}$.

40

235

167

68

235

172
76.17

23.83

100.00

64

9

1212

27.27

72.73

100.00

86.59

13.41

100.00

195

17.02

100.00

71.06

28.94

100.00

73.19
$907 \quad 79.63$

$232 \quad 20.37$

$1139 \quad 100.00$ 


$\begin{array}{llrrrr}\text { Verb im Plural } & 63 & 26.81 & 402 & 35.29 & \\ \text { Samplegröße } & 235 & 100.00 & 1139 & 100.00 & \\ 18 \text { Verb im Präsens } & 154 & 65.53 & 766 & 67.25 & 0.51 \\ \text { Verb in anderen Tempora } & 81 & 34.47 & 373 & 32.75 & \\ \text { Samplegröße } & 235 & 100.00 & 1139 & 100.00 & \\ 19 \text { Verb im Indikativ } & 216 & 91.91 & 1013 & 88.94 & +1.35 \\ \text { Verb in anderen Modi } & 19 & 8.09 & 126 & 11.06 & \\ \text { Samplegröße } & 235 & 100.00 & 1139 & 100.00 & \\ 20 \text { Verb im Aktiv } & 229 & 97.45 & 1104 & 96.93 & +0.43 \\ \text { Verb im (W-/S-)Passiv } & 6 & 2.55 & 35 & 3.07 & \\ \text { Samplegröße } & 235 & 100.00 & 1139 & 100.00 & \\ & & & & & \end{array}$

Das Grundsample B, gebildet auf der Grundlage des Theaterstücks "Die Fische" von Peter Hacks und den oben erwähnten Diskussionen "Fragen der Verkehrssicherheit", "Schulklassengespräch mit Günter Grass" sowie "Moral 71. Zum Beispiel Abtreibung", enthält 244 Sätze und satzwertige Konstruktionen, die Nominalisierungen mit Satzgliedcharakter enthalten. Die Nominalisierungen im Grundsample B unterlagen anderen Auswahlkriterien als die Nominalisierungen des Grundsamples A (vgl. 3.13.9). Das Grundsample B wurde mit dem Kontrollsample B verglichen. Das Kontrollsample B enthält 1415 Sätze und satzwertige Konstruktionen ohne Nominalisierungen mit Satzgliedcharakter.

\section{TABELLE 2: VERGLEICH GRUNDSAMPLE B MIT KONTROLLSAMPLE B}

$\begin{array}{lccc}\text { DIE ENTSPRECHENDE } & \text { GRUNDSAMPLE } & \text { KONTROLLSAMPLE } & I_{\mathrm{d}} \\ \text { VORHERSAGE } & \text { Werte } \% & \text { Werte } & \%\end{array}$

\section{A1. ZUGRUNDELIEGENDES VERB (GS)}

$\begin{array}{lrr}\text { 1. kompliziert gebautes Verb } & 167 & 61.85 \\ \text { einfach gebautes Verb } & 103 & 38.15 \\ \text { Samplegröße } & 270 & 100.00 \\ \text { 2. langes Verb <36 Silben> } & 176 & 65.19 \\ \text { kurzes Verb <12 Silben> } & 94 & 34.81 \\ \text { Samplegröße } & 270 & 100.00 \\ \text { 3. } & 179 & 66.30 \\ \text { seltenes Verb } & 91 & 33.70 \\ \text { Säufiges Verb + unklar } & 270 & 100.00\end{array}$

A2. KERN DER NOMINALISIERUNG (GS): KERN DER SUBSTANTTVGRUPPE (KS)

$\begin{array}{lrr}\text { 4. } \text { kompliziert gebautes Subst. } & 234 & 86.67 \\ \text { einfach gebautes Substantiv } & 36 & 13.33 \\ \text { Samplegröße 270 } & 100.00 & \end{array}$


5. selten vorkommendes Subst. $\quad 231 \quad 85.56$

häufiges Subst. + unklar $\quad 39 \quad 14.44$

Samplegröße $270 \quad 100.00$

6. bedeutungsenger als Verb

$140 \quad 51.85$

$135 \quad 50.00 \quad+0.43$

nicht bedeutungsenger als $\mathrm{V}$

$130 \quad 48.15$

$135 \quad 50.00$

Samplegröße

$270 \quad 100.00$

$270 \quad 100.00$

B1. NOMINALISIERUNG (GS) : SUBSTANTIVGRUPPE / BASISVERBPHRASE (KS)

7. weniger Ergänzungen $<0>$ als Verbalphrase im Satz mehr Ergänzungen <1-4> als Verbalphrase im Satz Teilsamplegröße pronominalisierte Subst. Samplegröße

8. Subjektgenitiv belebt Subjektgenitiv unbelebt Samplegröße 31

9. Objektgenitiv unbelebt Objektgenitiv belebt Samplegröße

10 Nom. als direktes Objekt Nom. als anderes Satzglied Samplegröße

11 NP als Thema des Satzes NP als Thema des Satzes Samplegröße
$157 \quad 60.62$

$102 \quad 39.38$

$259 \quad 100.00$

11

270

$\begin{array}{ll}22 & 70.97\end{array}$

$9 \quad 29.03$

100.00

$\begin{array}{ll}42 & 80.77\end{array}$

$10 \quad 19.23$

$52 \quad 100.00$

$46 \quad 17.04$

$224 \quad 82.96$

$270 \quad 100.00$

$35 \quad 24.48$

$108 \quad 75.52$

$143 \quad 100.00$

B2. KONTEXT DER NOMINALISIERUNG : KONTEXT DER SUBSTANTIVGRUPPE

12 häufiger in Hauptsätzen 132 57.39 846 63.61 $-1.80$ seltener in Nebensätzen

98
42.61 $484 \quad 36.39$

Teilsamplegröße $230 \quad 100.00$

$1330 \quad 100.00$

Infinitivsätze

Partizipialphrasen

Samplegröße

13 in NS mit infinitem Verb in NS mit finitem Verb Samplegröße

$\begin{array}{rrrrr}14 & 12.50 & 85 & 14.94 & -0.67 \\ 98 & 87.50 & 484 & 85.06 & \\ 112 & 100.00 & 569 & 100.00 & \\ 115 & 87.12 & 704 & 83.22 & +1.13 \\ 17 & 12.88 & 142 & 16.78 & \\ 132 & 100.00 & 846 & 100.00 & \end{array}$


15 häufiger in bejahenden S. seltener in verneinenden $S$. Samplegröße

16 Verb in dritter Person

Verb in nicht-dritter Pers.

Samplegröße

17 Verb im Singular

Verb im Plural

Samplegröße

18 Verb im Präsens

Verb in anderen Tempora

Samplegröße

19 Verb im Indikativ

Verb in anderen Modi

Samplegröße

20 Verb im Aktiv

Verb im (W-/S-) Passiv 22

Samplegröße

$\begin{array}{rrrrr}202 & 87.83 & 1134 & 85.26 & +1.02 \\ 28 & 12.17 & 196 & 14.74 & \\ 230 & 100.00 & 1330 & 100.00 & \\ 192 & 83.48 & 978 & 73.53 & +3.22 \\ 38 & 16.52 & 352 & 26.47 & \\ 230 & 100.00 & 1330 & 100.00 & \\ 177 & 76.96 & 893 & 67.14 & +2.96 \\ 53 & 23.04 & 437 & 32.86 & \\ 230 & 100.00 & 1330 & 100.00 & \\ 171 & 74.35 & 983 & 73.91 & +0.14 \\ 59 & 25.65 & 347 & 26.09 & \\ 230 & 100.00 & 1330 & 100.00 & \\ 207 & 90.00 & 1201 & 90.30 & 0.14 \\ 23 & 10.00 & 129 & 9.70 & \\ 230 & 100.00 & 1330 & 100.00 & \\ 208 & 90.43 & 1248 & 93.83 & -1.91 \\ 9.57 & 82 & 6.17 & & \\ 230 & 100.00 & 1330 & 100.00 & \end{array}$

Diagramm 1: Statistischer Vergleich Grundsample A und Kontrollsample A

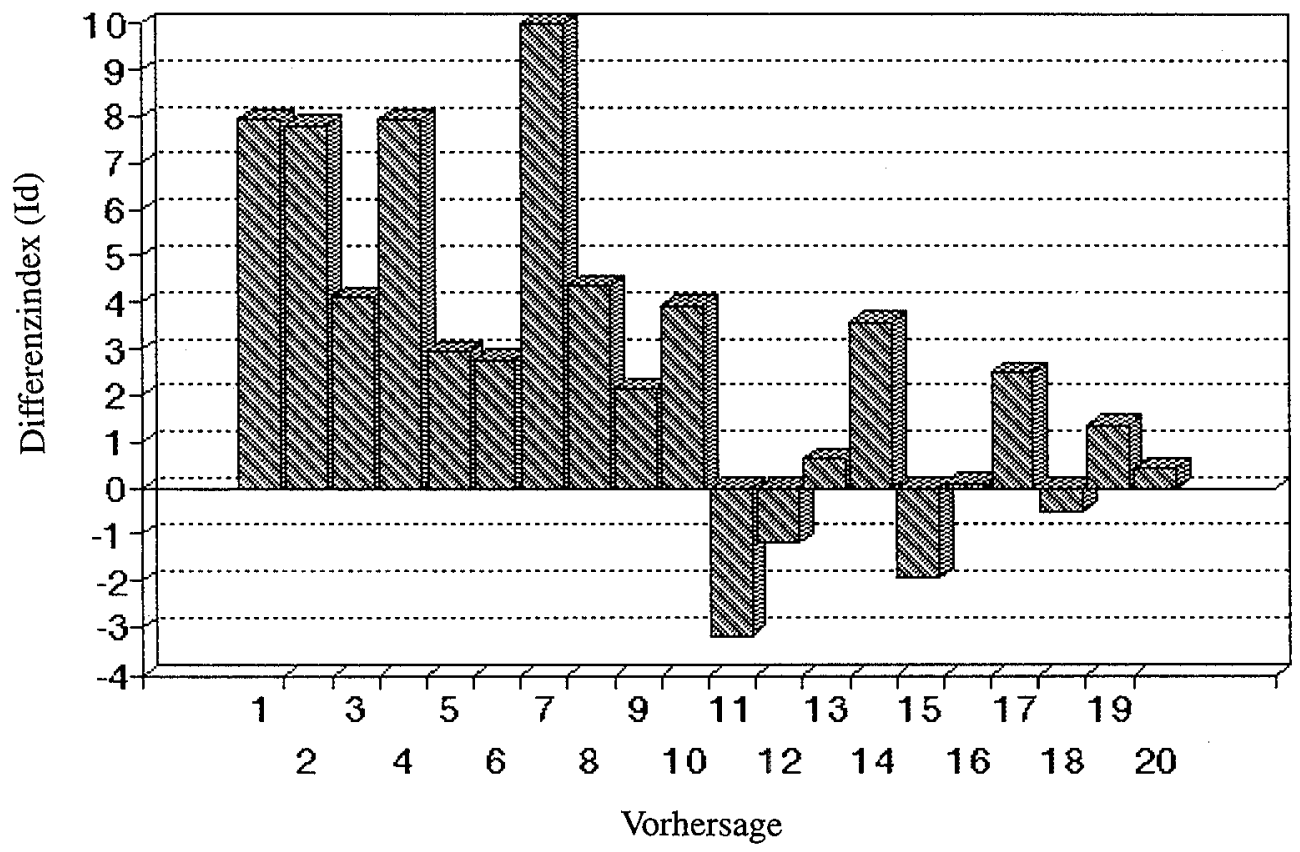


Diagramm 2: Statistischer Vergleich Grundsample B und Kontrollsample B

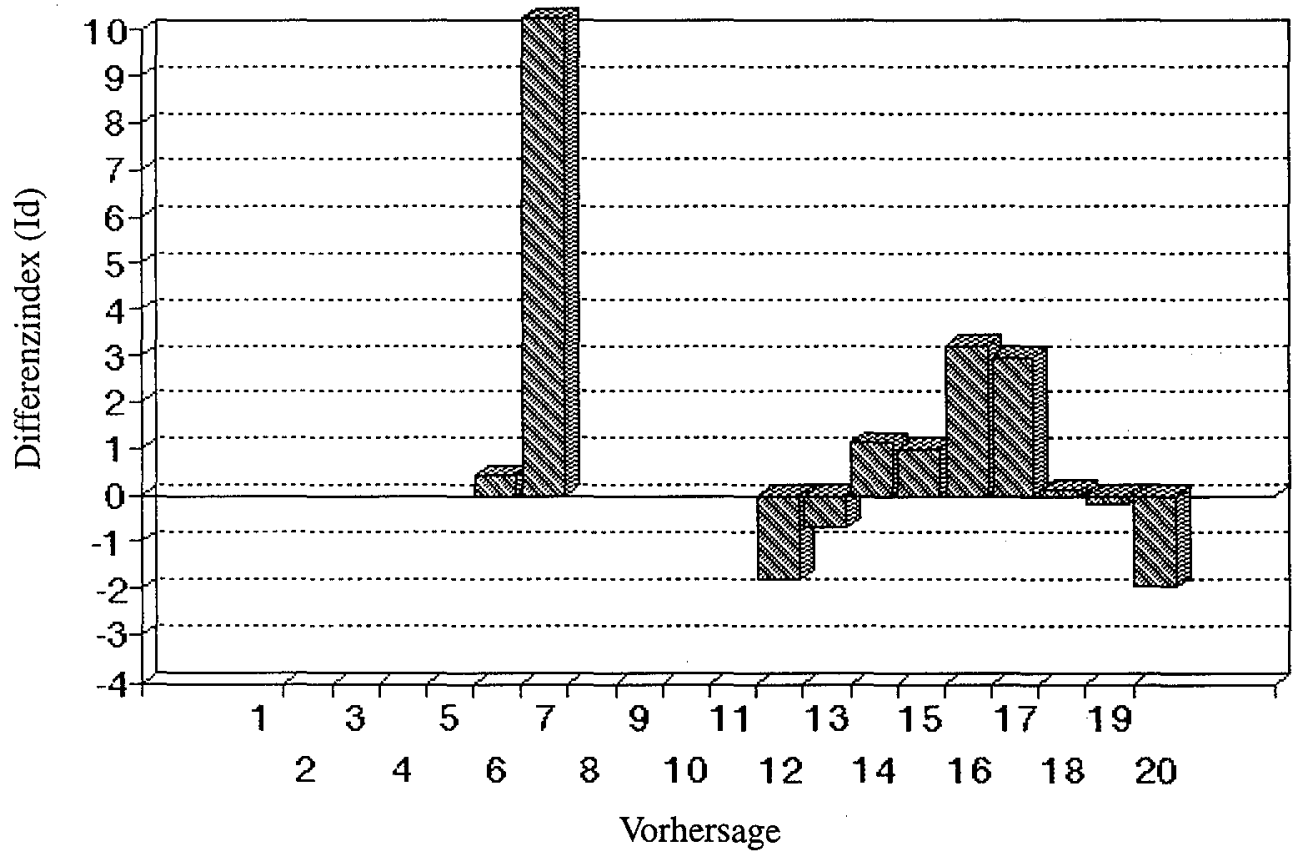

TABELLE 3: Der Anteil der Ableitungsaffixe in den Nominalisierungskernen der beiden Grundsamples $A$ und $B$.

\begin{tabular}{lrrrr} 
ABLEITUNGSSUFFIX & Werte & $\%$ & Werte & \multicolumn{1}{c}{$\%$} \\
{$[0]$} & 88 & 32.00 & 48 & 17.78 \\
{$[\mathrm{e}]$} & 20 & 7.27 & 13 & 4.81 \\
{$[\mathrm{en}]$} & 36 & 13.09 & 36 & 13.33 \\
{$[(\mathrm{er}) \mathrm{ei}]$} & 2 & .73 & 7 & 2.59 \\
{$[\mathrm{Ge}(\mathrm{e})]$} & 6 & 2.18 & 2 & .74 \\
{$[\mathrm{nis}]$} & 9 & 3.27 & 0 & .00 \\
{$[(\mathrm{~s}) \mathrm{t} / \mathrm{d}(\mathrm{e})]$} & 10 & 3.64 & 5 & 1.85 \\
{$[$ tum] } & 3 & 1.09 & 2 & .74 \\
{$[(\mathrm{at}) \mathrm{ion}]$} & 1 & .36 & 9 & 3.33 \\
{$[$ ung] } & 100 & 36.36 & 148 & 54.81 \\
VSOTA & 275 & 100.00 & 270 & 100.00
\end{tabular}




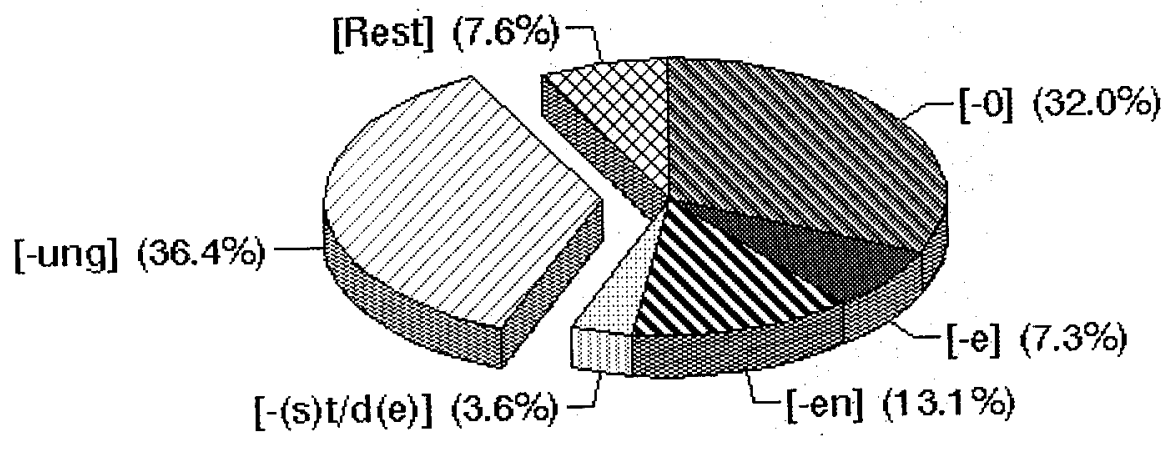

Diagramm 4: Ableitungstypen Grundsample B

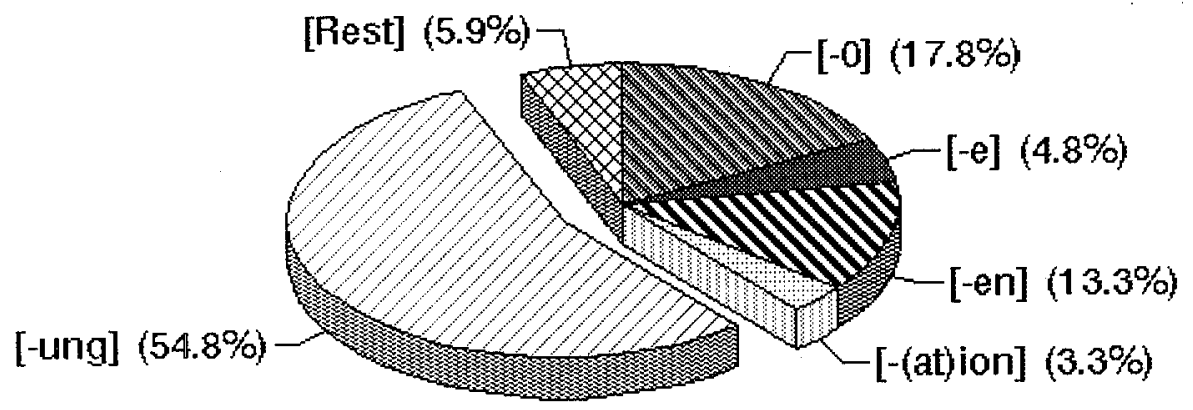




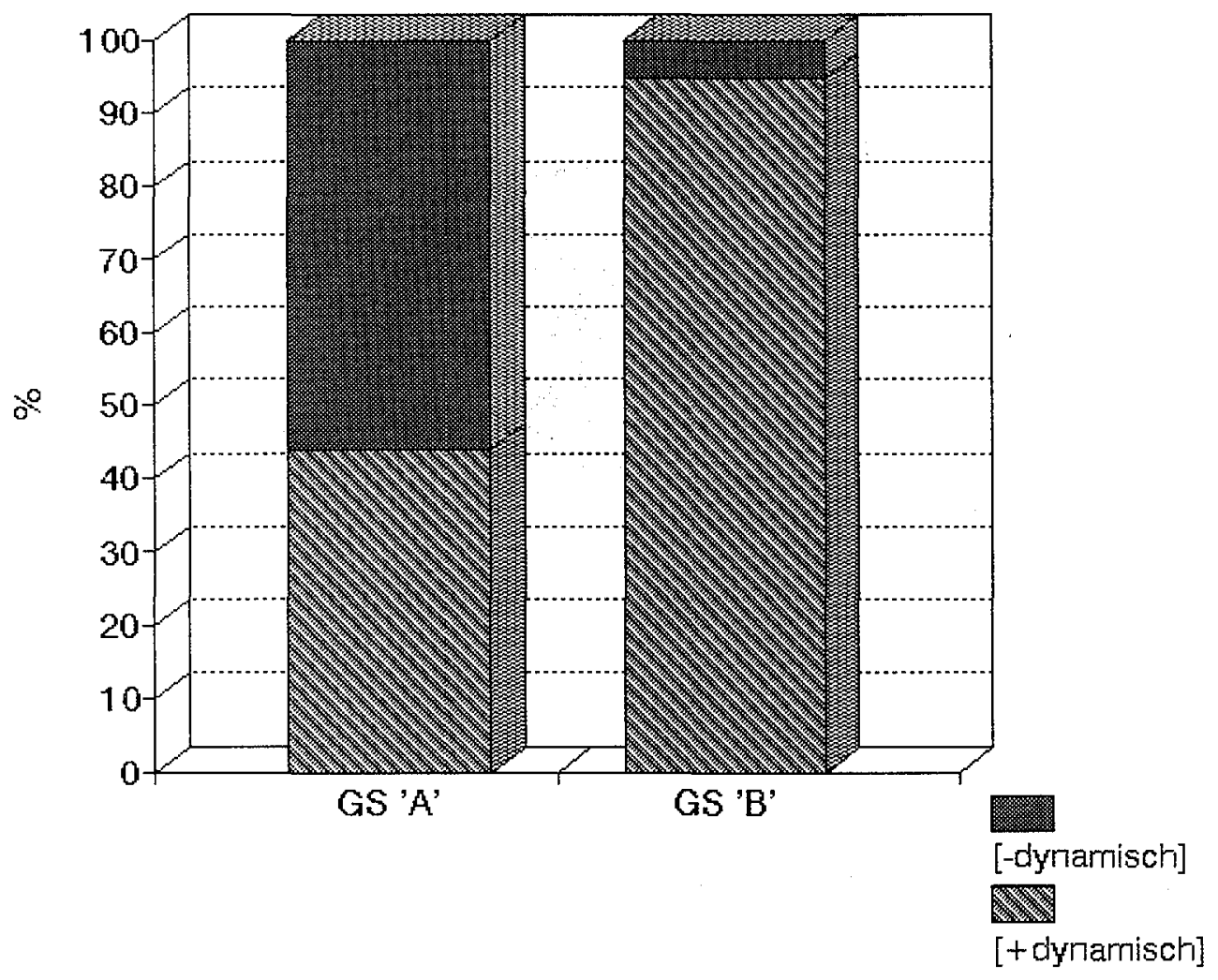

Im Vergleich zwischen Grundsample A und Kontrollsample A sind von 20 (=100 $\%)$ geprüften Vorhersagen

- 12 Vorhersagen $(=60 \%)$ signifikant bestätigt,

-7 Vorhersagen ( $35 \%$ ) nicht signifikant und

-1 Vorhersage $(=5 \%)$ ist signifikant nicht bestätigt (vgl. Tabelle 1 und das dazugehörige Diagramm 1).

Im Vergleich zwischen Grundsample B und Kontrollsample B sind von 11 (= 100 \%) geprüften Vorhersagen

-3 Vorhersagen $(=27 \%)$ signifikant bestätigt und

-8 Vorhersagen $(=73 \%)$ nicht signifikant

(vgl. Tabelle 2 und das dazugehörige Diagramm 2).

Vergleicht man jene Vorhersagen miteinander, die sowohl anhand Grundsample A als auch anhand Grundsample B geprüft wurden (vgl. Tabelle 2 und Diagramm 2), dann ergibt sich für den Vergleich Grundsample A : Kontrollsample A das Verhältnis $4: 7: 0$ 
(d.h. 4 Vorhersagen sind signifikant bestätigt, 7 Vorhersagen sind nicht signifikant und keine Vorhersage ist falsifiziert).

Im Hinblick auf jene 9 Vorhersagen, die den Kontext der Nominalisierungen betreffen (vgl. Vorhersage 12 - 20 in Tabelle 1, 2 und in den Diagrammen 1 und 2), ergibt sich sowohl für den Vergleich Grundsample A : Kontrollsample A als auch für den Vergleich Grundsample B : Kontrollsample B das Verhältnis $2: 7: 0$ (d.h. 2 Vorhersagen sind signifikant bestätigt, 7 Vorhersagen sind nicht signifikant und keine Vorhersage ist falsifiziert).

Die Mehrheit der geprüften Vorhersagen konnte somit bestätigt werden, nur eine Vorhersage (Opposition Thema : Rhema) wurde mit statistischer Signifikanz verworfen. Größer als erwartet ist der Anteil der nicht signifikanten Vorhersagen zum syntaktischen Verhalten der Nominalisierungen (vgl. die Vorhersagen 7-20 in Tabelle 1 bzw. die Vorhersagen 7, 12-20 in Tabelle 2). Allerdings tendieren die meisten der nicht signifikanten Differenzindices in die von uns vorhergesagte Richtung (vgl. Diagramm 1 und 2). Die vorgeführten Ergebnisse der Untersuchung werten wir als vielversprechend und im folgenden Sinn überzeugend: Wenn die behandelten grammatischen Parameter keinen Einfluß auf den Gebrauch der Nominalisierungen ausüben würden, müßte die statistische Lage in beiden Grund- und Kontrollsamples ungefähr dieselbe sein.

\section{BEMERKUNGEN ZU EINIGEN STATISTISCHEN ERGEBNISSEN}

Die Gründe, warum einige Vorhersagen in unserem Korpus nicht bestätigt worden sind, sind uns zum Teil bekannt (vgl. auch Orešnik/Snedec/Teržan/Trobevšek-Drobnak 1990: 9-11 und Orešnik 1990b: 94-97). Die unten folgenden Annahmen betreffen die Textauswahl (5.1), die Auswahl der Kontrollsamples (5.2), die Auswahl der Grundsamples (5.3), die Auswahl und Markiertheit von Parametern (5.4) sowie die Verstärkungsund Schwächungshypothese selbst (5.5).

5.1 Unsere Vorhersagen gelten in erster Linie für Texte, in denen die von der Markiertheitstheorie angenommen Häufigkeiten der Markiertheitswerte realisiert sind. Theoretisch treten die markierten Werte der grammatischen Parameter in Texten seltener auf als die unmarkierten bzw. weniger markierten. Solche Texte nennen wir 'Idealtexte'. Ob es sie wirklich gibt, muß offen bleiben. Wir vermuten allerdings, daß natürliche Gesprächsabläufe dem Ideal nahekommen. Außerdem sollten die Texte für die statistische Berechung hinreichend lang und homogen sein sowie ausreichend einschlägige Beispiele der untersuchten Konstruktion liefern. Die Texte, die wir für unser Korpus ausgewählt haben, erfüllen zwar grob gesehen die genannten Forderungen, aber dennoch sind einige Abstriche zu machen. 
Das Drama "Die Fische" (vgl. 4) ist eigentlich kein natürlicher Gesprächsablauf, sondern ein simulierter (stilisiert wirkender). Außerdem wird der dramatische Dialog stellenweise durch etwas längere Erzählpartien (Monologe) unterbrochen. Die statistischen Verhältnisse werden durch diese Eigenheiten sicher beeinflußt.

Im Kontrollsample B stimmt z.B. der Anteil der Nebensätze oder der Anteil der passivischen Sätze zwar im groben mit der Markiertheitstheorie überein, ist jedoch höher als erwartet:

- Hauptsatz : Nebensatz $\rightarrow 846: 484(63,6 \%: 36,4 \%),{ }^{21}$

- Aktivsatz : Passivsatz $\rightarrow 1248: 82(93,8 \%: 6.2 \%)$.

Zum Vergleich sollen die entsprechenden Anteile im Kontrollsample A dienen, die in Einklang mit unseren Erwartungen über natürliche Gesprächsabläufe stehen:

- Hauptsatz : Nebensatz $\rightarrow 907: 232(79,6 \%: 20,4 \%)$,

- Aktivsatz : Passivsatz $\rightarrow 1104: 35$ (96.9\%: 3.1\%).

Die größeren Anteile im Kontrollsample B stehen im Zusammenhang mit der darin dominierenden Textsorte, denn drei der vier Texte, die als Datenbasis dienen, gehören zu den Diskussionen (vgl. 4). Die meisten der darin auftretenden Sprecher sind zu den dort gewählten Themenkreisen routiniert vorbereitet, sprechen daher oft nicht spontan, verlegen die Hauptinformationen häufig in die Nebensätze, während sie in den einleitenden Hauptsätzen vor allem den Sprechakt bzw. ihre Einstellungen näher bestimmen (vgl.das Beispiel (5.1)).

z.B. (5.1) Ich glaube, das würde eine sehr lange Diskussion hier hervorrufen. (Fragen der Verkehrssicherheit, S. 96)

Der relativ häufigere Gebrauch des Passivs hängt mit der Themenwahl (Verkehrssicherheit, Abtreibung, Schriftstellerei) zusammen. Das Agens der Handlung ist dabei oft irrelevant und fällt daher weg.

Unsere Textauswahl stand vor dem Dilemma, möglichst natürliche Gesprächsabläufe und gleichzeitig möglichst viele Nominalisierungen zu finden. Diese beiden Forderungen scheinen einander zu widersprechen, denn für den Gebrauch von Nominalisierungen muß ein ziemlich hohes Stilniveau vorausgesetzt werden; Nominalisierungen sind nämlich typisch für bestimmte Funktionalstile (z.B. Zeitungssprache, Wissenschaftssprache), die wohl nicht zu den natürlichen Gesprächsabläufen gehören. Unsere Textauswahl stellt also einen Kompromiß zwischen Natürlichkeits- und Stilniveauforderung dar.

5.2 Ein direkter Vergleich zwischen geschwächter und entsprechender nichtgeschwächter Konstruktion (hier: zwischen Nominalisierung und entsprechender Satzkonstruktion ${ }^{22}$ ) ist nicht immer möglich. Man kann daher gezwungen sein, die statisti- 
sche Lage bei den geschwächten Konstruktionen mit der Lage generell außerhalb der geschwächten Konstruktion vergleichen zu müssen. Ein indirekter Vergleich kann sich jedoch auf die Bestätigung einer Annahme ungünstig auswirken.

Dies scheint im Vergleich zur Opposition Thema : Rhema und zu den Annahmen bezüglich der Nominalisierungskontexte eben der Fall gewesen zu sein (vgl. Tabelle 1 bzw. Tabelle 2 und die dazugehörigen Diagramme 1 und 2).

Die Nominalisierungskerne, d.h. die Verbalabstrakta, gehören der Bedeutung nach zu den kompliziertesten Substantiven. Ihre komplizierte Bedeutung scheint sich auch in der syntaktischen Konstruktion der Nominalisierung niederzuschlagen. Wir nehmen daher an, daß Nominalisierungen im Vergleich zu den im Kontrollsample auftretenden Substantivgruppen, in denen Substantive oder Pronomina als Gliedkern vorkommen, markierter sind. Wir betrachten also die Markiertheitsrelation: $m(\operatorname{PrnP}, \mathrm{NP}, \mathrm{SP})^{23}$ als guiltig.

Als Anzeichen dafür, daß diese Annahme richtig sein könnte, sehen wir folgende Daten aus unserem statistischen Vergleich:

1. Nominalisierungen im Grundsample A (insbesondere solche mit Verbalabstraktum auf [-ung] als Kern) traten im Vergleich zu den Nominalphrasen im Kontrollsample A signifikant häufiger mit Rechtsattributen auf (vgl. auch Sommerfeldt 1969:291). Wir nehmen dabei an, daß Rechtsattribute markierter sind als Linksattribute. ${ }^{24}$ Angenommene Markiertheitsrelation: m (Linksattr., Rechtsattr.).

2. Nominalisierungen im Grundsample A traten im Vergleich zu den Nominalphrasen im Kontrollsample A häufiger als Rhema und (nichtsignifikant) auch ausgeklammert auf (vgl. auch Petry 1988: 69f,87).

3. Enthielt eine Nominalisierung in Grundsample A oder B ein Verbalabstraktum auf [-ung] als Gliedkern, dann erschien es im Vergleich zu den Substantivgruppen in den Kontrollsamples A und B signifikant häufiger in Nebensätzen. ${ }^{25}$

22 Bisher haben wir sowohl Haupt- wie Nebensätze als mögliche syntaktische Varianten von Nominalisierungen gesehen. Weiter unten sowie in 5.4.3 wird diese Annahme in dem Sinne abgewandelt, daß Nebensätze (bzw. Gliedsätze) und Nominalisierungen als syntaktische Varianten zu werten sind.

23 NP ist eine Substantivgruppe mit Substantiv als Kern, PrnP ist eine Substantivgruppe mit Pronomen als Kern und SP ist eine Substantivgruppe mit Verbalabstraktum als Kern.

24 Mit Rechtsattributen sind hier vor allem Genitivattribute wie Subjekt- und Objektgenitive, Präpositionalattribute und satzförmige Attribute gemeint. Typische Linksattribute sind Adjektive, Partizipien und Possessivpronomina.

25 Für die Verbalabstrakta auf [-ung] aus dem Grundsample A beträgt der Differenzindexwert $I_{d}=$ -2.28 , für die Verbalabstrakta auf [-ung] aus dem Grundsample $B$ hingegen $I_{d}=-2.67$. Die beiden Werte sind signifikant und negativ. Sie bestätigen unsere Vorhersage also nicht. Der Anteil der Hauptsätze im Grundsample A mit [-ung]-Nominalisierungen beträgt $69.3 \%$, der entsprechende Anteil im Grundsample B hingegen 53.4\%. Der Anteil der Hauptsätze im Kontrollsample A beträgt $79.6 \%$, im Kontrollsample B hingegen 63.6\%. Der Anteil der Hauptsätze im Grundsample B, die ein 
Die Nichtbestätigung der Vorhersage zur Opposition Thema : Rhema (vgl. Tabelle 1) ist wohl auf den indirekten Vergleich zurückzuführen. Wir haben nämlich Nominalisierungen mit Substantivgruppen aus dem Kontrollsample (vgl. die Kriterien unter 3) verglichen, anstatt sie direkt mit Gliedsätzen (den entsprechenden nichtgeschwächten Konstruktionen) zu vergleichen. Unsere Vorhersage wäre somit bestätigt worden, wenn wir die Nominalisierungen hinsichtlich der Opposition Thema : Rhema mit Gliedsätzen verglichen hätten. ${ }^{26}$ Nominalisierungen sind nämlich ein Mittel zur Thematisierung von Satzinhalten und als Nominalphrasen im Satz wesentlich beweglicher als Gliedsätze.

Auch die Nicht-Signifikanz bzw. Nichtbestätigung der Vorhersagen bezüglich der Nominalisierungskontexte können auf den indirekten Vergleich zurückgeführt werden. Wäre ein direkter Vergleich mit den entsprechenden Gliedsätzen möglich, wären unsere Annahmen vielleicht bestätigt worden.

5.3 Unsere Vorhersagen gelten für dasjenige Stadium in der Entwicklung der Nominalisierungen, das der Entstehungsphase folgt und ihrer Lexikalisierung vorausgeht. Möglicherweise wurden unsere Kriterien zur Auswahl von Nominalisierungen dieser Forderung nicht gerecht.

Anzeichen dafür sind vielleicht die Eigenschaften der Nominalisierungen mit Verbalabstraktum auf [-ung] als Gliedkern. Sie unterscheiden sich nämlich z.T. signifikant von denen anderer Nominalisierungen in den beiden Grundsamples:

So scheinen Verbalabstrakta auf [-ung] häufiger mit Rechtsattributen (insbesondere Objektgenitiven) aufzutreten als andere Verbalabstrakta. Nominalisierungen mit Rechtsattributen scheinen jedoch im Sätzen häufiger als Rhema vorzukommen und wegen Produktions- und Dekodierungsproblemen häufiger ausgeklammert zu werden (vgl. Petry 1988:69f,87).

Die Verbalabstrakta auf [-ung] in den beiden Grundsamples kommen außerdem signifikant häufiger in Nebensätzen ${ }^{27}$ vor als die Substantivgruppen in den beiden Kontrollsamples.

Diese Tendenzen treffen für die übrigen Ableitungen nicht oder im geringeren Maße zu (d.h. sie sind nicht signifikant). Die Trennung von produktiven und nicht-produktiven Ableitungsmustern in unserer Untersuchung war möglicherweise nicht scharf genug (vgl. Tabelle 3 und die Tortendiagramme 3 und 4 und die aufgelisteten Beispiele

Verbalabstraktum mit Objektgenitiv enthalten, beträgt sogar nur $47 \%$.

26 Unter den Nominalisierungen in den Hauptsätzen des Grundsamples A haben wir $22.2 \%$ als Thema eingeordnet. Unter den abhängigen Sätzen mit finitem oder infinitem Verb, die Satzglieder der Hauptsätze des Kontrollsamples A sind, waren nur $15.4 \%(143=100 \%)$ nach unserer Analyse Thema der Hauptsätze. Der Unterschied ist wegen des kleinen Kontrollsamples zwar nicht signifikant, zeigt aber Tendenz zur Bestätigung unserer Annahme.

27 Als Nebensätze gelten in dieser Untersuchung alle finiten abhängigen Sätze mit V-Letzt-, V2- oder V1-Stellung. 
unter 3). Der relativ hohe Anteil der lexikalisierten Ableitungen in den Grundsamples ist möglicherweise schuld daran, daß mehrere Vorhersagen zum Kontextverhalten der Nominalisierungen nicht bestätigt werden konnten, denn wir nehmen an, daß lexikalisierte (bzw. sich lexikalisierende) Nominalisierungen sich grammatisch bereits (fast) wie andere Substantivgruppen (mit prototypischeren Substantiven) verhalten. ${ }^{28}$

5.4 Ein weiterer Grund, warum einige Vorhersagen nicht bestätigt worden sind, könnte auch der Umstand sein, daß die Markiertheitstheorie nicht in allen Punkten stimmt. Diese Schwierigkeit haben wir zu umgehen versucht, indem wir uns in unserer Untersuchung auf Markiertheitswerte beschränkten, von denen wir glaubten, sie seien relativ unumstritten. Jedoch ist es durchaus möglich, daß wir in dieser Hinsicht nicht genügend kritisch waren.

Als Beispiel mag uns die Opposition Hauptsatz : Nebensatz dienen. Im Zusammenhang mit ihr sehen wir drei verschiedene Hypothesen über die möglichen Markiertheitsverhältnisse:

5.4.1 In unserer Untersuchung haben wir die Auffassung übernommen, daß Nebensätze markierter sind als Hauptsätze, denn Nebensätze haben typischerweise eine syntaktische Position mehr als Hauptsätze (eine subordinierende Konjunktion nämlich).

Die Verbalabstrakta auf [-ung] in den beiden Grundsamples traten wider Erwarten häufiger in Nebensätzen auf, womit unsere Vorhersage, die sich nach der Markiertheitsrelation $\mathrm{m}$ (HS, NS) richtete, verworfen wurde.

5.4.2 Es gibt jedoch auch stichhaltige Gründe, das Gegenteil anzunehmen, nämlich daß Hauptsätze markierter sind als Nebensätze, denn in Nebensätzen ist der Anteil bestimmter als einfach bzw. unmarkiert geltender Konstruktionen (z.B. der Pronomina in Subjektfunktion oder nicht-satzförmiger Objekte und Prädikative) größer als in Hauptsätzen (vgl. dazu Orešnik 1990a). Außerdem scheinen Nebensätze (wie auch Nominalisierungen) nicht über die illokutive Kraft der Hauptsätze zu verfügen, was man als weiteres Merkmal der größeren Markiertheit von Hauptsätzen werten könnte. In diesem Falle gälte also die umgekehrte Markiertheitsrelation: m (NS, HS).

Richteten wir uns nach der Markiertheitsrelation m (NS, HS), wäre unsere Vorhersage zur Opposition Hauptsatz : Nebensatz zwar bestätigt, aber dieses Ergebnis würde sich nicht gut mit den anderen in 5.3 genannten Eigenschaften der Verbalabstrakta auf [-ung] decken.

5.4.3 Weiterhin kann man sich auf den Standpunkt stellen, daß Haupt- und Nebensätze nur dann miteinander vergleichbar sind, wenn sie syntaktische Varianten darstellen (z.B. kausale denn-Sätze : weil-Sätze).

Mit dem Prozeß der Lexikalisierung von Verbalabstrakta geht auch die Tendenz ihrer semantischen Weiterentwicklung einher. Neben einigen Bedeutungsvarianten, die Verbalabstrakta von ihren zugrundeliegenden Verben übernommen haben, entwickeln sich oft neue (nominale) Bedeutungsvarianten (z.B. Ergebnisbezeichnung). 
Haupt- und Nebensätze ${ }^{29}$ haben zwar viele gemeinsame Eigenschaften, scheinen sich aber grundsätzlich dadurch zu unterscheiden, daß erstere typischerweise über illokutive Kraft verfügen, während letzteren diese Kraft nicht bzw. in einem geringeren Maße eigen ist. Hauptsätze kommen (wegen ihrer illokutiven Kraft) selbständig vor, Nebensätze treten hingegen prototypisch als Einbettungen in Matrixsätzen auf, sind also nur Satzglieder in Hauptsätzen (bzw. in übergeordneten Sätzen) und kommen nicht selbständig vor. ${ }^{30}$

Wenn wir unseren Blick auf andere Konstruktionen ausweiten, können wir feststellen, daß auch Infinitivsätze, satzwertige Partizipialkonstruktionen und Nominalisierungen in der Regel ohne illokutives Potential sind. ${ }^{31}$ Ebenso wie Nebensätze treten sie prototypisch als Einbettungen in Matrixsätzen auf, sind also nur Satzglieder in Hauptsätzen (bzw. in übergeordneten Sätzen) und kommen nicht selbständig vor.

Daher ist es wohl nicht abwegig, Gliedsätze ${ }^{32}$ als die entsprechenden nichtgeschwächten syntaktischen Varianten der Nominalisierungen anzusehen. Sie unterscheiden sich jedoch durch ihre grundsätzlich verschiedene Rolle in der Thema-RhemaGliederung der Äußerung (siehe 5.2).

Besonders gut vergleichbar sind Nominalisierungen mit den passivischen finiten Gliedsätzen sowie Infinitivsätzen und Partizipialkonstruktionen mit Satzgliedcharakter, denn in den genannten Konstruktionen ist es möglich, Ergänzungen auszusparen. Besonders häufig ist der Wegfall des Agens der Handlung (Agensabgewandtheit). Die Nominalisierungen bieten gegenüber den passivischen man-Aktivsätzen, den Infinitivsätzen und den satzwertigen Partizipialkonstruktionen sowie den passivischen (finiten) Gliedsätzen den Vorteil, daß der Kern der Konstruktion (das Verbalabstraktum) leichter zu handhaben ist (man denke insbesondere an Nominalblöcke mit bedeutungsschwachen, relativ häufig auftreten Verben) und zugleich hohen stilistischen Ansprüchen genügen kann sowie eine weitreichende Variation des Ausdrucks erlaubt. Angenommene Markiertheitsrelation: m (SP, IS/PK, NS) ${ }^{33}$

Nebensätze, die von verba dicendi (im weiten Sinne) abhängen, haben zwar syntaktisch gesehen den Status von Ergänzungssätzen, verfügen jedoch über illokutives Potential. Das wird auch daran deutlich, daß in ihnen alle Modalpartikeln auftreten können, die auch in Hauptsätzen auftreten können.

30 Es gibt jedoch Sätze mit der Verbstellung, wie sie in Nebensätzen vorkommt (also Verb-LetztStellung), die selbständig auftreten und auch über illokutives Potential verfügen. In solchen Sätzen sind daher auch Modalpartikeln möglich. Solche Sätze sind wohl meist nicht als Nebensätze oder elliptisch verwendete abhängige Sätze zu bezeichnen.

31 Zur Ähnlichkeit von Nominalisierungen und Nebensätzen hinsichtlich der illokutiven Nicht-Selbständigkeit siehe Zimmermann 1986:203.

32 Relativsätze werden hier außer Acht gelassen, da wir in unseren Grundsamples nur mit Nominalisierungen als Satzgliedern $z u$ tun haben.

33 Mayerthaler/Fliedi/Winkler (1991) stufen Infinitivsätze hingegen gegenüber finiten Nebensätzen als markiertere Konstruktionen ein. 
5.5 Gilt die umgekehrte Annahme, daß geschwächte Konstruktionen vorzugsweise in komplizierten Umgebungen statt in einfachen Umgebungen auftreten, und setzt man außerdem die Markiertheitsrelation $\mathrm{m}$ (HS, NS) voraus, dann kann man dazu zumindest zwei weitere Annahmen machen:

5.5.1. Nominalisierungen sind nach unserer Annahme geschwächte syntaktische Konstruktionen. Entgegen unseren Erwartungen treten die Nominalisierungen mit Verbalabstraktum auf [-ung] jedoch häufiger in den markierteren Nebensätzen auf. Als Grund könnte man außer den in 5.2 und 5.3 genannten eine Tendenz zur (teilweisen) Kompensierung der durch die syntaktische Schwächung auftretende Bedeutungsverdunkelung und daraus resultierenden Verständnisschwierigkeiten vermuten, also eine der Schwächung entgegenwirkende Tendenz. Die Nominalisierungen würden demzufolge in eine markiertere grammatische Umgebung "versetzt", um den Verstehensprozeß weiterhin zu gewährleisten.

5.5.2. Nominalisierungen sind geschwächte syntaktische Konstruktionen und sind ein Mittel zur Vereinfachung der Äußerungsstruktur. In der deutschen Gegenwartssprache wirkt eine Tendenz zur Linearisierung der Satzstruktur (Admoni 1973:79f). Diese Tendenz bewirkt eine Zunahme von Substantivgruppen und Parataxe und gleichzeitig eine Abnahme der Hypotaxe. Besonders stark ist der zahlenmäßige Rückgang von Nebensätzen zweiten und höheren Grades (vgl. Beneš 1981:185f). Wir könnten daher annehmen, daß die Tendenz zur Linearisierung der Satzstruktur in Nebensätzen stärker wirkt als in Hauptsätzen, besonders stark jedoch in Nebensätzen, von denen ein oder mehrere weitere Nebensätze abhängig sind.

z.B. (5.2) Sie müssen in Ihrer Argumentation, wenn Sie die ersatzlose STREICHUNG des Paragraphen 218 durchsetzen wollen, klartun, daß der Gesetzgeber in diesem Falle nicht die Pflicht hat, das werdende Leben als Bestandteil unseres Lebens insgesamt zu schützen. (Moral 71. Zum Beispiel Abtreibung, S. 369)

(5.3) Sie müssen in Ihrer Argumentation, wenn Sie es durchsetzen wollen, daß der Paragraph 218 ersatzlos gestrichen wird, klartun, daß der Gesetzgeber in diesem Falle nicht die Pflicht hat, das werdende Leben als Bestandteil unseres Lebens insgesamt zu schützen.

Die Einebnung einer unübersichtlichen, komplexen Äußerungsstruktur durch Nominalisierung von Nebensätzen (zweiten und höheren Grades) erleichtert dem Sprecher das Produzieren von inhaltlich komplexen Äußerungen. Dem Hörer bzw. Leser liegt zwar eine einfachere (linearere) Äußerungsstruktur vor, aber dafür muß er mehr Denkarbeit investieren, um die meist fehlenden Ergänzungen und nur implizit oder im Kontext der Nominalisierungen ausgedrückten syntaktischen Beziehungen zu erschließen. Sind die Nominalisierungen nicht zu umfangreich und nicht zu dicht gestaffelt, leidet der Verstehensprozeß gewöhnlich nicht. Zumindest in Gesprächsabläufen kann das Fehlende meist relativ leicht aus dem Kontext bzw. aus dem Weltwissen erschlossen werden. Daher stellt eine verzweigte hypotaktische Struk- 
tur wohl ein größeres Dekodierungsproblem dar als eine geradlinigere Äußerungsstruktur mit einer Nominalisierung.

Gestört wird dieses Balancespiel zwischen optimaler Sparsamkeit im Ausdruck und optimaler Deutlichkeit im Ausruck allerdings in einigen Funktionalstilen (z.B. in der Gesetzessprache, Publizistik, Wissenschaftssprache), in denen sich die Nominalisierungen häufen und mit zahlreichen und komplexen Attributen überfrachtet werden. Diese Funktionalstile sind jedoch nicht zu den natürlichen Gesprächsabläufen zu zählen. Die angenommenen Markiertheitsverhältnisse sind hier zum Teil wahrscheinlich nicht gültig. Eine Häufung komplexer Nominalisierungen dürfte jedenfalls für den Schreiber/Sprecher ein größeres Kodierungs- und für den Leser/Hörer ein wohl noch größeres Dekodierungsproblem darstellen als komplexe mehrstufige hypotaktische Satzstrukturen.

\section{ZUSAMMENFASSUNG}

Viele Germanisten glauben, in der deutschen Sprache eine allgemeine Tendenz zur Nominalisierung feststellen zu können. Bekanntlich zeigt sich diese Neigung in vielen Literatursprachen der Welt. Der Gebrauch von Nominalisierungen statt expliziterer finiter Sätze ist keine neue sprachliche Erscheinung, weder im Deutschen noch in anderen Sprachen. So war diese Möglichkeit bereits im Althochdeutschen durchaus gegeben (Heringer 1968:120). Der Gebrauch von Substantivgruppen, insbesondere von Nominalisierungen, begann aber in der zweiten Hälfte des 19. Jahrhunderts (vor allem in einigen Funktionalstilen) stark zuzunehmen. Auch im 20. Jahrhundert gewinnen die Nominalisierungen weiter an Boden. Diese Tendenz steht in weitgehendem Zusammenhang mit der gleichzeitigen Abnahme im Gebrauch des Satzgefüges. Die Tendenzwende vom explizit-hypotaktischen Stil zum komprimierenden Nominalisierungsstil läßt sich aus der politischen und wirtschaftlichen Geschichte des Landes erklären.

Die Nominalisierung der Prädikation hat in der deutschen Gegenwartssprache zwei verschiedene Ausprägungen:

1. zum einen als Funktionsverbgefüge,

2. zum anderen als freie Fügungen.

1. Bei der Bildung von Funktionsverbgefügen (FVG) wird ein Verb in mehrere Wörter 'aufgespalten'. Die Bedeutung einer solchen Fügung ist dabei meist enger bzw. spezialisierter als die des entsprechenden einfachen Verbs. FVG sehen wir als Periphrasen für einfache Verben (Adjektive sollen hier unberücksichtigt bleiben) an. Periphrasen jedoch halten wir im Vergleich zu den entsprechenden einfachen Verben für verstärkte Konstruktionen. (dieser Blickrichtung wurde in Petrič 1991 und 1994 Beachtung geschenkt). 
2. Die Bestandteile der 'freien Fügungen' (den Ausdruck haben wir von Köhler 1985 übernommen) sind im Gegensatz zu den FVG semantisch und syntaktisch selbständiger und nicht fest aneinander gebunden. Als selbständige Einheiten in freien Fügungen begegnen uns Nominalisierungen (mit Verbalabstrakta als deren Kern). Durch Nominalisierung wird der Prädikatsausdruck komprimiert, womit auch eine Verdunkelung des (Satz-)Inhalts einhergeht. Daher sehen wir Nominalisierungen im Vergleich zu den entsprechenden finiten Sätzen als geschwächte syntaktische Konstruktion an. Der Kern einer Nominalisierung ist hingegen im Vergleich zum entsprechenden Verbalstamm eine verstärkte Wortbildungskonstruktion.

Um die beiden zuletzt genannten Annahmen (Punkt 2) zu bestätigen bzw. zu widerlegen, haben wir anhand von vier Stichproben (zwei Grundsamples und zwei Kontrollsamples) die aus den o.g. Annahmen abgeleiteten Vorhersagen (vgl. 2.1-20) statistisch geprüft (vgl. Abschnitt 4).

Während die Annahme, daß die Gliedkerne der Nominalisierungen (d.h. die Verbalabstrakta) im Vergleich zu den entsprechenden Verbstämmen verstärkte Wortbildungskonstruktionen sind, voll bestätigt werden konnte, sind die Ergebnisse im syntaktischen Bereich weniger eindeutig.

Obwohl im syntaktischen Bereich im Vergleich zwischen Grundsample A und Kontrollsample A nur eine Vorhersage (Opposition Thema : Rhema) verworfen und sechs Vorhersagen bestätigt wurden, stimmt die relativ hohe Zahl der nicht signifikanten Ergebnisse (sieben Vorhersagen) nachdenklich. Die nicht signifikanten Ergebnisse betreffen den Kontext der Nominalisierungen.

Auch im Vergleich zwischen Grundsample B und Kontrollsample B, der uns in dieser Hinsicht Klarheit verschaffen sollte, ergab ein ähnliches Bild: Von den neun Vorhersagen, die den Kontext der Nominalisierungen betreffen, sind nur zwei Vorhersagen bestätigt, während die Unterschiede zwischen den beiden Stichproben in den übrigen sieben Fällen nicht signifikant sind.

Die Nicht-Signifikanz einiger statistischer Ergebnisse bzw. die teilweise NichtBestätigung im syntaktischen Bereich haben wir mit der Textauswahl (5.1), der Auswahl der Kontrollsamples (5.2), der Auswahl der Grundsamples (5.3), der Problematisierung von Markiertheitswerten (5.4) sowie mit umgekehrten Annahmen der Verstärkungs- und Schwächungstheorie (5.5) in Zusammenhang gebracht und zu erklären versucht.

Die Datenbasis ist leider zu schmal, um die offengebliebenen Fragen mit Gewißheit beantworten zu können. Einige unserer Zweifel könnte ein größeres Korpus unter Einbeziehung mehrerer Textsorten wahrscheinlich aus dem Wege räumen.

Aufgrund unserer statistischen Ergebnisse nehmen wir an, daß Nominalisierungen mit Nebensätzen (d.h. vor allem mit Gliedsätzen) vergleichbar sind, nicht hingegen mit Hauptsätzen, denn mit den Nebensätzen teilen sie die Eigenschaft, in der Regel illokutiv nicht selbständig verwendet werden zu können. Wir betrachten also Ne- 
bensätze (vor allem Gliedsätze) als die entsprechenden nichtgeschwächten (markierten) syntaktischen Varianten von Nominalisierungen.

Häufig ist der Gebrauch von Nominalisierungen motiviert durch die Absicht des Sprechers, bestimmte Argumente der Prädikation (z.B. das Agens) auszusparen. Dies rückt die Nominalisierungen in die Nähe der passivischen Nebensätze sowie der Infinitivsätze und satzwertigen Partizipialkonstruktionen.

Nominalisierungen (vielleicht nur Nominalisierungen mit nicht-lexikalisierten [-ung]-Verbalabstrakta) scheinen komplizierter zu sein als Substantivgruppen, die kein Verbalabstraktum als Gliedkern enthalten. Darauf scheinen die Ergebnisse zur ThemaRhema-Opposition und das häufigere Vorkommen der Verbalabstrakta auf [-ung] in Nebensätzen zu weisen.

Wir nehmen mit Rücksicht auf unsere statistischen Ergebnisse folgende Markiertheitsverhältnisse an: ${ }^{34}$

m (PrnP, NP, SP, PK, IS, NS).

Trotz einiger (noch) nicht bestätigter Vorhersagen im syntaktischen Bereich erscheint uns eine Übertragung der Annahme von der Existenz verstärkter und geschwächter Konstruktionen auf die Syntax sinnvoll.

\section{TEXTE}

HACKS, PETER (1981): Die Fische, S.169-231 in Ausgewählte Dramen Band 3. Aufbau Verlag. Berlin, Weimar 1981.

HEUTIGES DEUTSCH II/1 (1971): Texte gesprochener deutscher Standardsprache I. IDS Mannheim Forschungsstelle Freiburg i. Br. Max Hueber Verlag München / Pädagogischer Verlag Schwann. Düsseldorf 1971.

HEUTIGES DEUTSCH II/2 (1974): Texte gesprochener deutscher Standardsprache II. IDS Mannheim Forschungsstelle Freiburg i. Br. Max Hueber Verlag München / Pädagogischer Verlag Schwann. Düsseldorf 1974.

\section{LITERATUR}

ADMONI, WLADIMIR (1973): Die Entwicklungstendenzen des deutschen Satzbaus von heute. Linguistische Reihe Band 12. Max Hueber Verlag. München 1973.

PrnP ist eine Substantivgruppe mit pronominalem Gliedkern, NP ist eine Substantivgruppe mit nominalem Kern, SP ist eine Nominalisierung mit Verbalabstraktum als Gliedkern, IS steht für Infinitivsatz, PK für satzwertige Partizpialkonstruktion und NS für Nebensatz. 
BENEŠ, EDUARD (1981): Die formale Struktur der wissenschaftlichen Fachsprachen in syntaktischer Hinsicht. In: BUNGARTEN, THEO (Hg.) (1981): Wissenschaftssprache. Wilhelm Fink Verlag. München 1981. S. 185-212.

ten CATE, ABRAHAM P. (1985): Aspektualität und Nominalisierung. Zur Bedeutung satzsemantischer Beziehungen für die Beschreibung der Nominalisierung im Deutschen und im Niederländischen. Peter Lang. Frankfurt a.M. 1985.

DUDEN (19761981): Das große Wörterbuch der deutschen Sprache in sechs Bänden. Bibliographisches Institut. Mannheim 1976-1981.

ENGEL, ULRICH (1988): Deutsche Grammatik. Julius Groos Verlag. Heidelberg 1988.

ENGEL, ULRICH / SCHUMACHER, HELMUT (1978): Kleines Valenzlexikon deutscher Verben. TBL Verlag Gunter Narr. Tübingen 1978.

FLEISCHER, WOLFGANG (1983): Wortbildung der deutschen Gegenwartssprache. VEB Bibliographisches Institut. Leipzig 1983.

HAVERS, WILHELM (1931): Handbuch der erklärenden Syntax. Ein Versuch zur Erforschung der Bedingungen und Triebkräfte in Syntax und Stilistik. Heidelberg 1931.

HELBIG, GERHARD (1986): Zu umstrittenen Fragen der substantivischen Valenz. In: Deutsch als Fremdsprache 1986/4, S. 200-207.

HELBIG, GERHARD / BUSCHA, JOACHIM (1987): Deutsche Grammatik. Ein Handbuch für den Ausländerunterricht. VEB Verlag Enzyklopädie. Leipzig 1987.

HERINGER, HANS-JÜRGEN (1968): Die Opposition von 'kommen' und 'bringen' als Funktionsverben. Sprache der Gegenwart Band III. Pädagogischer Verlag Schwann. Düsseldorf 1968.

KÖHLER, CLAUS (1985): Verben in deutschsprachigen Fachtexten - Supplementverben (eine Voraussetzung der Nominalität von Fachtextsätzen). Schriftenreihe Fachsprache - Fremdsprache - Muttersprache, TU Dresden, Heft 1.

MAYERTHALER, WILLI (1980): Morphologische Natürlichkeit. Athenaion. Wiesbaden 1980 .

MAYERTHALER / FLIEDL / WINKLER (1991): Infinitivprominenz in europäischen Sprachen. Projektbericht. Universität Klagenfurt, Institut für Sprachwissenschaft. Klagenfurt 1991.

MEIER, HELMUT (1978): Deutsche Sprachstatistik (in zwei Bänden). Georg Olms Verlag. Hildesheim, New York 1978.

OREŠNIK, JANEZ (1990a): Main vs. Subordinate Clauses: Simple or Complex? In: Razprave - Dissertationes XIII, Razred za filološke in literarne vede - Classis II: Philologia et Litterae, Slovenska akademija znanosti in umetnosti - Academia Scientarium et Artium Slovenica. Ljubljana 1990.

OREŠNIK, JANEZ (1990b): Periphrasen sind verstärkte Konstruktionen. In: Boretzky N./Enninger W./ Stolz T. (1990) (Hgg.): Spielarten der Natürlichkeit Spielarten der Ökonomie. Beiträge zum 5. Essener Kolloquim über "Grammatikalisierung: Natürlichkeit und Systemökonomie" vom 6.10.-8.10.1988 an der Uni- 
versität Essen. Zweiter Band, erster Halbband, S. 85-99. Universitätsverlag Norbert Brockmeyer. Bochum 1990.

OREŠNIK, JANEZ / SNEDEC, ANDREJ / TERŽAN, KARMEN / TROBEVŠEKDROBNAK, FRANČIŠKA (1990): Introduction to the Subsequent Three Papers in the Present Volume. In: LINGUISTICA XXX, S. 5-12. Ljubljana 1990.

PAVLIĆ, I. (1985): Statistička teorija i primjena. Tehnička knjiga. Zagreb 1985.

PETRIČ, TEODOR (1990): Posamostaljenja v knjižni nemščini (Nominalisierungen im Standarddeutschen. Magistrsko delo (Magisterarbeit). Filozofska fakulteta (Philosophische Fakultät). Ljubljana 1990.

PETRIČ, TEODOR (1991): Funktionsverbgefüge als verstärkte syntaktische Konstruktionen). Manuskript eines Referats anläßlich der 19. Arbeitstagung österreichischer Linguisten (Klagenfurt, 25.-27.10.1991).

PETRIČ, TEODOR (1994): Funktionsverbgefüge und Nominalisierungsverbgefüge im Deutschen als verstärkte syntaktische Konstruktionen im Vergleich zu stammgleichen Verben und Adjektiven. In: Papiere zur Linguistik Nr. 2/1994:125-150.

PETRY, ULRIKE (1988): Zur kommunikativen Wirkung syntaktischer Strukturen. Eine Untersuchung am 7. "Bundesgenossen" Johann Eberlins von Günzburg. In: BRANDT, W. (Hg.) (1988): Sprache in Vergangenheit und Gegenwart. Marburger Studien zur Germanistik Band 9. Hitzeroh. Marburg 1988.

PETZ, BORIS (1985): Osnovne statističke metode za nematematičare. SNL. Zagreb 1985.

SANDBERG, BENGT (1982): Zur Valenz der Substantive. Deutsch als Fremdsprache 1982/5, S. 272-279.

SCHÄUBLIN, PETER (1972): Probleme des adnominalen Attributs. Morpho-syntaktische und semantische Untersuchungen. Studia Linguistica Germanica 5. Walter de Gruyter Berlin New York 1972.

SOMMERFELDT, KARL-ERNST (1969): Zur Struktur der Substantivgruppe in einigen funktionalen Stilen. In: Deutsch als Fremdsprache 1969/5, S. 287-295.

SOMMERFELDT, KARL-ERNST / SCHREIBER, HERBERT (1983): Wörterbuch zur Valenz und Distribution der Substantive. VEB Bibliographisches Institut. Leipzig 1983.

TEUBERT, WOLFGANG (1979): Valenz des Substantivs - Attributive Ergänzungen und Angaben. Sprache der Gegenwart, Band IL. Pädagogischer Verlag Schwann. Düsseldorf 1979.

TOMAN, JINDŘICH (1987): Wortsyntax. Eine Diskussion ausgewählter Probleme deutscher Wortbildung. Max Niemeyer Verlag. Tübingen 1987.

WELLMANN, HANS (1975): Deutsche Wortbildung - Typen und Tendenzen in der Gegenwartssprache, 2. Hauptteil: Das Substantiv. Pädagogischer Verlag Schwann, Dïsseldorf 1975.

ZIMMERMANN, ILSE (1983): Untersuchungen zum Verhältnis von Substantivgruppe und Nebensatz. In: Studia grammatica XXII, S. 201-242. 


\section{POSAMOSTALJENJE KOT ZGLED OŠIBLJENIH SKLADENJSKIH ZGRADB}

Naša osnovna delovna domneva je, da se nekatere oblike in skladenjske zgradbe jezika vedejo nasproti nekaterim drugim (sopomenskim) oblikam in skladenjskim zgradbam kot ošibljene ali kot okrepljene oblike oz. zgradbe. Iz te okolnosti sledijo nekatere značilnosti vedenja takih oblik oz. zgradb.

Jedro posamostaljenja v nemščini je večinoma neka tvorjenka. Tvorjenke pa so glede na svojo besedotvorno podstavo okrepljene oblike. Posamostaljenje kot skladenjska zgradba je ošibljena zgradba glede na stavek z osebno glagolsko obliko.

Za prevero napovedi smo oblikovali po dva osnovna vzorca in po dva kontrolna vzorca. Jezikovno gradivo so nam bila štiri besedila z ustnimi dvogovori: Hacksova drama "Die Fische" in tri diskusije z naslovi "Meinung gegen Meinung. Fragen der Verkehrssicherheit", "Moral 71. Zum Beispiel Abtreibung" in "Schulklassengespräch mit Günter Grass".

Statistična primerjava osnovnega vzorca A s kontrolnim vzorcem A je dala naslednje statistično pomenljive izide: Jedra posamostaljenj so pretežno izpeljana iz "težkih" glagolov (besedotvorno zapletenih, dolgih in redko uporabljenih) in sodijo med najbolj zapletene samostalnike (besedotvorno zapletene, redko uporabljene in $\mathrm{z}$ ožjim pomenom kot izhodiščni glagoli). Posamostaljenja kot ošibljene skladenjske zgradbe vsebujejo manj dopolnil kot ustrezni izhodiščni glagoli v stavčni zgradbi, najpogosteje pa so brez dopolnil. Če je v prilastku posamostaljenja izražen kak stavčni člen izhodiščnega stavka, potem je to najpogosteje osebek, če gre za živo, in premi predmet, če gre za neživo. Posamostaljenja najpogosteje nastopajo kot premi predmet. Pogosteje nastopajo posamostaljenja $v$ izjavnih stavkih in $v$ stavkih $z$ glagolom $v$ ednini. Naše domneve ne potrjujeta razmerje med izhodišči in neizhodišči stavkov. Ostalih sedem obravnavanih slovničnih parametrov je statistično nepomenljivih.

S statistično pomenljivostjo imajo posamostaljenja osnovnega vzorca B manj dopolnil izhodiščnega glagola in se pojavljajo pogosteje $v$ stavkih $z$ glagolom $v$ tretji osebi ednine. Ostalih osem obravnavanih parametrov je statistično nepomenljivih.

Po našem mnenju je iz navedenih rezultatov razvidna težnja, da se pojavljajo posamostaljenja pogosteje $v$ preprostejšem slovničnem okolju kot $v$ bolj zapletenem ( $v$ primerjavi $z$ njihovimi neošibljenimi skladenjskimi različicami - odvisnimi stavki). Sklepamo, da lahko nauk o sistemskih okrepitvah in ošibitvah razširimo tudi na skladnjo. 